\title{
Interactions between axon-like projections extended by Drosophila Follicle Stem Cells dictate cell fate decisions
}

Eric H. Lee ${ }^{1,2^{*}}$, Daniel Zinshteyn ${ }^{1}$, Melissa Wang ${ }^{1,2}$, Jessica Reinach ${ }^{2}$, Cindy Chau $^{2}$, Kelly Costa ${ }^{2}$, Alberto Vargas ${ }^{1}$, Aminah Johnson ${ }^{1}$, Jennifer I. Alexander ${ }^{1,2}$, Alana M. O'Reilly ${ }^{1,2,3^{\star}}$

${ }^{1}$ Molecular Therapeutics Program, Fox Chase Cancer Center, Philadelphia, PA 19111, USA

${ }^{2}$ Immersion Science Program, Fox Chase Cancer Center, Philadelphia, PA 19111, USA

Contact:

Alana M. O’Reilly

Molecular Therapeutics Program

Fox Chase Cancer Center

Philadelphia, PA 19111

215-214-1653

* Corresponding authors

Alana M. O’Reilly: Alana.OReilly@fccc.edu

Eric H. Lee: Eric.Lee@fccc.edu

Characters: 41,692 


\section{Abstract:}

Stem cells cycle between periods of quiescence and proliferation to promote healthy tissue aging. Once proliferation is initiated, mechanisms that control the balance between self-renewal and differentiation must be engaged to ensure maintenance of stem cell pools until the next quiescent cycle occurs. Here, we demonstrate that dynamic axon-like projections extended by Follicle Stem Cells (FSCs) in the Drosophila ovary control the self-renewal-differentiation balance. Known axon growth regulators still life and sickie are necessary and sufficient for FSC projection growth, mediating organization of germline cyst architecture during follicle formation, controlling targeting of projections to FSCs or germ cells, and regulating expression of the cell fate determinants Eyes Absent (Eya) and Castor (Cas). Our results support a model in which FSC projections function similarly to axons, providing structural organization to a dynamic organ while mediating communication between distinct cell types to effect the key cell fate decision to self-renew or differentiate.

\section{Keywords:}

Stem cell, self-renewal, oogenesis, communication, axon-like, cytoplasmic projections, cell fate, follicle formation

\section{Introduction:}

The equilibrium between stem cell self-renewal and differentiation is a cornerstone of tissue health. Healthy, heterogeneous stem cell pools must be maintained throughout the lifetime of the animal, while also producing the differentiated daughter cells necessary for optimal tissue function (Goodell \& Rando, 2015; Greulich \& Simons, 2016). Controlled shifts mediated by changes in signals that promote selfrenewal versus differentiation may be leveraged for tissue repair after injury or prevention of aging symptoms (Goodell \& Rando, 2015; Xin, Greco, \& Myung, 2016). In contrast, continuous imbalance can lead to aberrant states such as tumor formation when self-renewal is favored, or stem cell loss when differentiation is the primary outcome. Defining the molecular mechanisms that determine stem cell fate is therefore a pressing need.

Housed in microenvironments called niches, stem cells rely on their surroundings for signals and nutrients that enable and promote the properties of self-renewal and differentiation (Xin et al., 2016). In cases like well-studied germline stem cells (GSCs) in Drosophila, signals from the niche confer near-immortal status, ensuring a long functional lifespan of individual GSC clones and inheritance of stem cell function through generations (Hinnant, Merkle, \& Ables, 2020). Other stem cells exist in an aggressive, competitive environment, where limited niche space drives selection of stem cells in the right time and place to self-renew, with losers of the competition displaced to undergo differentiation (Albert Hubbard \& Schedl, 2019; Clevers \& Watt, 2018; Nelson, Chen, \& Yamashita, 2019; Rust \& Nystul, 2020).

Recent evidence points to proliferation rates as key for competitive edge in stem cell niches, with higher rates of proliferation associated with retention (Amoyel, Simons, \& Bach, 2014; de Navascués et al., 2012; Greulich \& Simons, 2016; Hsu et al., 2017; Jin et al., 2008; Kirilly, Spana, Perrimon, Padgett, \& Xie, 2005; Kronen, Schoenfelder, Klein, \& Nystul, 2014; Reilein, Melamed, Tavaré, \& Kalderon, 2018; Snippert et al., 2010; Su, Nakato, Choi, \& Nakato, 2018). Over time, stem cells with even a slight proliferative advantage can take over the niche, resulting in a clonal stem cell population and elimination of the initial heterogenous pool (Greulich \& Simons, 2016). This drift toward clonality is directly associated with loss of stem cell function and tissue aging in multiple stem cell populations, with significant work focused on development of approaches to maintain heterogeneity as a strategy to promote healthy aging (Haas, Trumpp, \& Milsom, 2018; Wahlestedt et al., 2017). Emerging evidence strongly suggests that imposing non-proliferative, resting states equalizes stem cells within a pool, reducing the effects of a slight proliferative advantage and promoting fair competition upon re-initiation of proliferation (Cho et al., 2019; Greulich \& Simons, 2016; Urbán, Blomfield, \& Guillemot, 2019; van Velthoven \& Rando, 2019). 
Nutrient restriction promotes stem cell quiescence, with periods of fasting and feeding regulating the proliferation status of stem cell pools and maintenance of heterogeneity through the aging process (Ables, Laws, \& Drummond-Barbosa, 2012; Bruens et al., 2020; Hartman, Strochlic, Ji, Zinshteyn, \& O'Reilly, 2013; Laws \& Drummond-Barbosa, 2016; Schultz \& Sinclair, 2016; Spehar, Pan, \& Beerman, 2020; van Velthoven \& Rando, 2019). The ability to manipulate stem cell pools through diet presents an exceptional opportunity to define cellular processes involved in quiescence to proliferation transitions and to uncover molecular mechanisms that may lead to development of intervention strategies to promote stem cell heterogeneity and healthy aging.

Follicle Stem Cells (FSCs) in the Drosophila ovary are an example of the competitive stem cell paradigm (Nystul \& Spradling, 2007, 2010; Reilein et al., 2017; Reilein et al., 2018). Exquisitely feeding-dependent, FSCs undergo quiescence to proliferation transitions, utilize proliferative advantage for long-term retention, and drift toward clonality over time (Drummond-Barbosa \& Spradling, 2001; Greulich \& Simons, 2016; Hartman et al., 2013; Hsu et al., 2017; Kirilly et al., 2005; Kronen et al., 2014; Reilein et al., 2018; Snippert et al., 2010; Song \& Xie, 2003; Su et al., 2018; X. Wang \& Page-McCaw, 2014; Zhu A. Wang, Huang, \& Kalderon, 2012; Z. A. Wang \& Kalderon, 2009). Hedgehog (Hh) signaling translates feeding status to control FSC quiescence to proliferation transitions(Hartman et al., 2013). Hh is released from terminal filament and cap cells (apical cells) located at the extreme apical end of the germarium, the stem cell compartment of the fly ovary (Figure 1A), in response to cholesterol ingestion (Çiçek et al., 2016; Hartman et al., 2013). Hh accumulation within FSCs correlates precisely with proliferation induction, a process that requires the Hh effectors Smoothened (Smo) and Cubitus Interruptus (Ci) (Hartman et al., 2013). Proliferating FSCs then undergo self-renewal and/or initiate differentiation into epithelial follicle cells (Margolis \& Spradling, 1995; Nystul \& Spradling, 2007; Reilein et al., 2017; Reilein et al., 2018). FSCs located in the center of the germarium at the Region 2A/2B border (also called Layer 2) have the highest propensity to self-renew (W. Dai, A. Peterson, T. Kenney, H. Burrous, \& D. J. Montell, 2017; Margolis \& Spradling, 1995; Nystul \& Spradling, 2007; Reilein et al., 2017; Reilein et al., 2018). Cells located one cell diameter to the anterior in Region 2A (Escort Cell/Layer 3) or posterior in Region 2B (Pre-Follicle Cell/Layer 1) also are capable of self-renewal, but exhibit a strong preference for differentiation into escort cells or follicle cells, respectively (Reilein et al., 2017) (Figure 1A). Follicle cells encapsulate 16-cell germline cysts, forming follicles (egg chambers) comprised of a single-layered cuboidal epithelium and a 16-cell germline cyst that develop synchronously through 14 stages of development to produce a mature egg (Figure 1A). Within the FSC pool, divisions are asynchronous, often with only one FSC dividing at a time (Reilein et al., 2017). Cells residing in Region 2A-B can differentiate into follicle cells without division (Reilein et al., 2018), suggesting that multiple mechanisms are employed to maintain a long-lived stem cell pool and produce sufficient functional daughter cells for normal development. Recent work demonstrates overlapping gene expression signatures and the ability to change position among cells in and near the FSC niche (Jevitt et al., 2020; Reilein et al., 2018; Slaidina, Banisch, Gupta, \& Lehmann, 2020), demonstrating plasticity among cellular residents in Region 2A-B. Despite these advances, the relationships between cell cycle entry, dynamic changes in morphology and position, and the self-renewal versus differentiation fate decisions of FSCs are not well understood.

Our previous work described axon-like projections present in FSCs in nutrient-restricted conditions that grow rapidly upon feeding (Hartman et al., 2015). We proposed that FSC projections might mediate communication between FSCs and cells that transit through the FSC niche. Analysis of the function of FSC projections has been challenging, due to the absence of genetic tools to separate projection growth from other FSC functions. Here, we identify the axon growth regulators still life (sif) and sickie (sick) as critical and specific regulators of FSC projection growth. We further demonstrate that proliferation and projection outgrowth are independent events, as proliferation is not sufficient for projection outgrowth and projections can elongate in the absence of proliferation. Finally, we demonstrate that FSC projections are required for egg chamber formation and preventing differentiation. Our results support a model in which FSC projections act to maintain the self-renewal versus differentiation equilibrium. 


\section{Results:}

Feeding independently regulates FSC proliferation and projection growth

The quiescence to proliferation transition of FSCs occurs rapidly, with release of $\mathrm{Hh}$ molecules from apical cells occurring as quickly as 15 minutes after feeding (Hartman et al., 2013). Hh accumulation in FSCs becomes detectable 3 hours after feeding, a timepoint that correlates with initiation of the quiescence to proliferation transition. FSC axonlike projections are prominent in steady state feeding conditions and 6 hours after feeding (Hartman et al., 2015). FSCs have two projections, a primary projection of $34.2+/-2.17 \mu \mathrm{m}$ in fed conditions, and a shorter, secondary projection of $16.8+/-2.28 \mu \mathrm{m}$, reminiscent of an axon-dendritelike organization (Figure 1B). The morphological similarity of FSC projections to axons suggested the possibility that projections might act to communicate signals that drive the transition into proliferation. To test this idea, we first created a timecourse to map the cellular events that occur during the FSC quiescence to proliferation transition (Figure 1C). Consistent with our previous work, FSC proliferation initiated between 3 and 6 hours after feeding, peaking at 6 hours and remaining elevated over the 24 hour timecourse (Figure 1C,D) (Hartman et al., 2013). FSC primary projections were short under nutrient-restricted conditions, exhibiting dramatic growth over the timecourse (Figure 1C,E,F) (Hartman et al., 2015). The timing of FSC projection growth was inconsistent with a significant role in inducing proliferation, however, as projection growth began between 6 and 12 hours after feeding, following the peak of the proliferative response (Figure 1C).

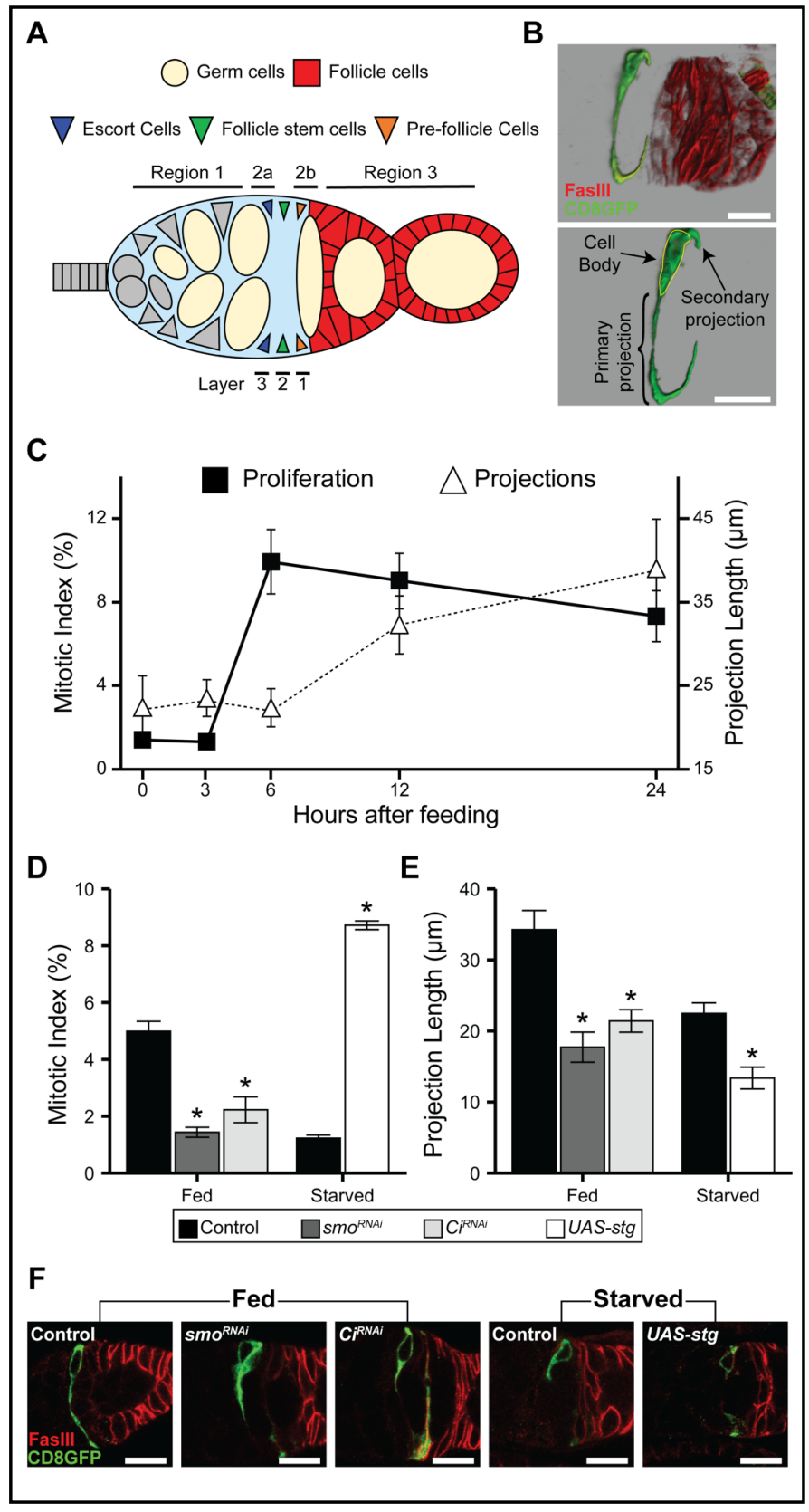

Figure 1. Hedgehog signaling regulates FSC proliferation and projection length.

(A) Schematic diagram of the germarium. FSCs (green) are located at the Region 2A/2B border, also called Layer 2. Cells in Region 2B/Layer1 (yellow, also called pre-follicle cells) preferentially produce follicle cells (red), but retain the capacity to self-renew or change position with the FSC niche. Cells in Region 2A/Layer 3 (blue), adopt an escort cell fate, but self-renew and generate follicle cells on rare occasions. Germline cysts (peach), interact with FSCs and become encapsulated by follicle cells to form egg chambers. Apical cells (gray rectangles), Germline Stem Cells (gray circles), Cystoblast (gray oval) and Inner Germarial Sheath (IGS/escort cells, gray triangles) are also shown in Region 1. (B) Representative image of FSC primary and secondary projections. Top, FSC (green), and follicle cells (red). Bottom, FSC only. Faslll (red) marks follicle cells. CD8GFP (green) marks FSCs and projections. (C) Time course of proliferation and projection extension. Flies were nutrient restricted (starved) prior to feeding of yeast $0,3,6,12$, and 24 hour timepoints after feeding are indicated. Frequency of germaria with at least one FSC in mitosis (PH3+) is shown. Projection length $(\mu \mathrm{m})$ was measured in MARCM GFP labeled FSCs at the indicated timepoints. (D) Quantification of proliferation frequency as measured by mitotic index (germaria containing PH3+ FSC/total), indicated for each genotype. Flies were nutrient-restricted (starved) for 3 days or fed for 6 hours after a 3 day nutrient restriction (fed). N>322. (E) Projection length $(\mu \mathrm{m})$ of MARCM GFP labeled primary projections in flies nutrient-restricted (starved) for 3 days or fed 6 hours after 3 days of nutrient restriction (fed). $N>6$. (F) Representative FSC projection images. CD8GFP (green) marks FSCs and projections. FasIII (red) marks follicle cells. ${ }^{*} p<0.01$ when compared to indicated control. (B,F) Scale bars are $10 \mu \mathrm{m}$. 
Proliferation and projection growth both depended on $\mathrm{Hh}$ signaling, with reduced expression of the $\mathrm{Hh}$ effectors smo or $\mathrm{Ci}$ in FSCs blocking the feeding response (Figure 1D-F) (Forbes, Lin, Ingham, \& Spradling, 1996; Hartman et al., 2010; Cynthia Vied \& Kalderon, 2009; Zhang \& Kalderon, 2001). This suggests two possibilities: 1) loss of Hh signaling in FSCs arrests cells in quiescence, with the resulting failure to induce sufficient proliferation preventing cellular events that occur later, or 2) the Smo-Ci cassette regulates proliferation and projection growth independently. If proliferation and projection growth are dependent events, we expected that induction of proliferation in nutrient restricted flies would trigger the entire quiescence to proliferation transition, including projection growth. Ectopic expression of the CDC25 homolog, string, is known to drive proliferation by activating cyclin-dependent kinases in S-phase and mitosis (Jimenez, Alphey, Nurse, \& Glover, 1990). We found that string expression in FSCs in nutrient-restricted flies promoted proliferation (Figure 1D), but further reduced projection length (Figure $1 \mathrm{E}, \mathrm{F})$. This suggests that feeding-dependent $\mathrm{Hh}$ signaling controls proliferation and projection growth independently, with proliferation occurring first, followed by projection growth.

\section{FSC projections exhibit homotypic and heterotypic interactions}

In steady state, continuously fed conditions, FSCs extend a $40 \mu \mathrm{m}$ axon-like projection across the germarium to the opposite side (Hartman et al., 2015) (Figure 1F). Previous work demonstrated formation of a weblike network of intertwined FSC projections that forms a barrier spanning the FSC niche at the Region 2A/2B border (Hartman et al., 2015). In addition, published work suggests that contact between FSC daughters and germ cells induces epithelial polarization as an early step in the differentiation process (Bhat et al., 1999; Bilder, Li, \& Perrimon, 2000; Goode, Melnick, Chou, \& Perrimon, 1996; Tanentzapf, Smith, McGlade, \& Tepass, 2000). Based on these observations, we predicted that FSC projections would form heterotypic, FSC-germline cyst (GC) interactions to promote induction of differentiation and formation of the follicular epithelium homotypic, as well as FSC-FSC interactions to create the niche-spanning barrier. To test this, we utilized the CoinFlp system to visualize boundaries between genetically marked interacting cells. The technique is based on GRASP, where two complementary parts of GFP (spGFP 1-10 and spGFP 11) are expressed on the plasma membrane of adjacent cells after a heat shock-dependent mitotic recombination event (Bosch, Tran, \& Hariharan, 2015). GFP is reconstituted only upon cell-cell interaction between opposing cells induced to express UAS-CD4-spGFP 1-10, under control of Actin-Gal4, and LexAop-CD4spGFP11, under control of Actin-LexGAD (Figure 2A) (Bosch et al., 2015). In fed conditions in control flies, the predominant interaction was FSC-FSC (57\%), with projections on adjacent FSCs exhibiting extensive overlapping interfaces (Figure 2B,E). 30\% of germaria exhibited heterotypic FSC-GC interactions, with projections completely surrounding cysts entering the plane of the FSC niche (Figure 2C,E). A small minority of FSCs (14\%), exhibited

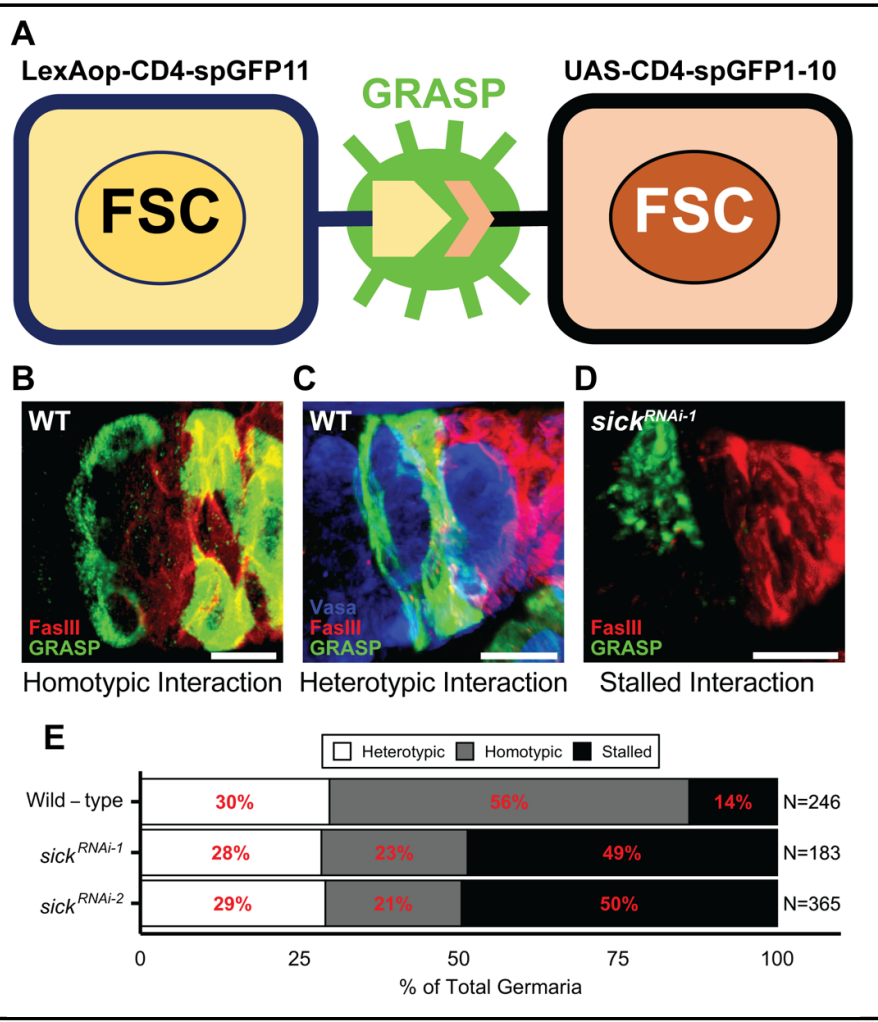

Figure 2: FSC projections exhibit homotypic and heterotypic interactions.

(A) Schematic of CoinFLP. After heat shock-induced mitotic recombination, one cell expresses exon 11 of split GFP fused to the transmembrane protein CD4 (CD4-spGFP11) under Actin-LexA control and a neighboring cell expresses exons 1-10 of split GFP fused to CD4 under Actin-Gal4 control. Transcellular binding reconstitutes the GFP, producing green fluorescence (GRASP). (B) Homotypic FSC-FSC interactions. FasIII (red) marks follicle cells. GRASP (green) marks FSCs and projections. C) Heterotypic interactions between FSC projections and germline cyst. (D) sick ${ }^{\mathrm{RNAi}}$ FSCs exhibit stalled projections. (E) Quantification of FSC projection interactions. $\mathrm{N}>183$. (B-D) Vasa (blue) marks germ cells. Fas III (red) marks follicle cells. GRASP (green) marks FSCs and projections. Scale bars are $10 \mu \mathrm{m}$. 


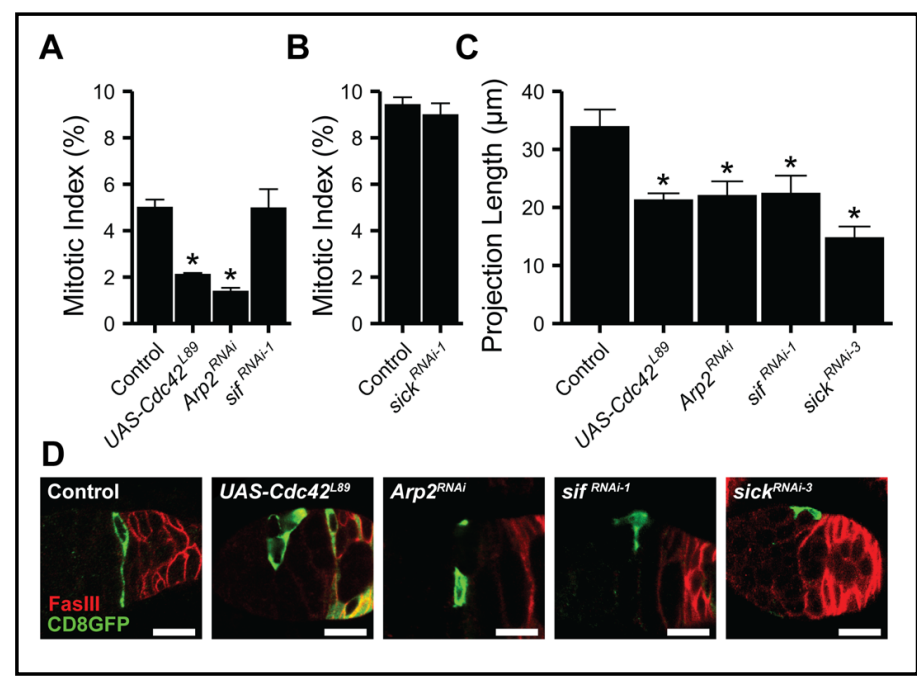

Figure 3: Candidate screen for FSC projection regulators. (A) FSC proliferation in control versus dominant negative or RNAi knockdown mutants, scored as mitotic index (germaria with $\mathrm{PH} 3+$ FSC/total). UAS-Cdc42 ${ }^{L 89}$ and $A r p 2^{J F 02585}$ values are from continuously fed flies. sif $^{R N A-1 i}$ values are from flies nutrient restricted for 3 days and then fed 6 hours. ${ }^{*} p<0.01$ when compared to $109-30$

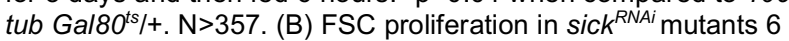
hours after feeding nutrient restricted flies. $N>509$. (C) Primary projection length in indicated genotypes. ${ }^{*} p<0.01$ when compared to 109-30 Gal4/+. N>6. (D) Representative images of projections. CD8GFP (green) marks FSCs and projections. FasIll (red) marks follicle cells. projections that failed to interact across the plane of the niche (Figure 2D,E), perhaps stalling or undergoing projection retraction or new growth. These results support the notion that FSC projections can interface with germline cysts passing through the niche or each other.

\section{Sif/TIAM-1 regulates FSC projections}

To date, we have identified three important regulators of projection length. The Hh effectors smo and $\mathrm{Ci}$ are necessary for projection growth during the quiescence to proliferation transition (Figure 1). In addition, integrin-mediated adhesion is necessary for directing projections around the surface of the germarium, a process linked with the orientation of FSC division and FSC anchoring to the germarium surface (Hartman et al., 2015). In all three cases, proliferation is dramatically reduced (Hartman et al., 2015; Hartman et al., 2010; O'Reilly, Lee, \& Simon, 2008; Zhang \& Kalderon, 2000), confounding interpretation of the impact of altering gene function specifically on FSC projections. To identify an FSC projectionspecific regulator, we took two approaches. First, we tested candidate genes with two key features: 1) known drivers of axon growth, that 2) act downstream of Smo to mediate Hh signaling (Drummond et al., 2018; Gallo, 2011; Sasaki, Kurisu, \& Kengaku, 2010). Of the genes that met this criteria, we found three with important roles in the FSC quiescence to proliferation transition. Reduced function of the small GTPase Cdc42 and the actin regulator Arp2 exhibited phenotypes overlapping with those seen in integrin, smo, or Ci mutants (Figure 3, 1D-F). The quiescence to proliferation transition was disrupted, with 3-4-fold lower FSC proliferation upon reducing Cdc42 or Arp2 function in FSCs (Figure 3A). Projection length also was dramatically shorter than in control flies (Figure 3C,D). FSCs detached from the basement membrane in both Cdc42 and Arp2 mutants, relocating to the center of the germarium (Figure 3D). This phenocopies integrin loss-of-function in FSCs (Hartman et al., 2015), but is not observed upon reduced expression of smo or Ci (Figure 1D). These results are consistent with an important role for dynamic actin regulation in promoting proliferation induction and subsequent projection growth during the quiescence to proliferation transition. Cdc42/Arp2 may function to mediate integrin signaling (Etienne-Manneville, 2004) or modulate Hh signaling directly (Drummond et al., 2018; Wan et al., 2013).

Smo binds directly to TIAM-1, a guanine nucleotide exchange factor that is known to activate downstream pathways to control neuronal protrusion and axon guidance (Demarco, Struckhoff, \& Lundquist, 2012; Kunda, Paglini, Quiroga, Kosik, \& Caceres, 2001; Mertens, Pegtel, \& Collard, 2006; Julian Ng \& Liqun Luo, 2004; Sasaki et al., 2010; Sone et al., 1997; Zheng, Diaz-Cuadros, \& Chalfie, 2016). Importantly, TIAM-1 is both necessary and sufficient for neurite extension in cultured mammalian neurons (Kunda et al., 2001), making it an attractive candidate for Hh-dependent regulation of FSC projection growth. Whereas reduced expression of still life (sif)(Sone et al., 1997), the fly homolog of TIAM-1, did not affect proliferation 6 hours after feeding (Figure 3A), FSC projections failed to grow (Figure 3C), indicating a key role for sif in regulation of projection growth following the quiescence to proliferation transition. 
sickie and still life are necessary and sufficient for FSC projection regulation

In addition to screening candidate Smo effectors, our second approach was to clone the gene associated with the 109-30 Gal4 driver. 109-30 Gal4 activates expression of genes under UAS control, with a high degree of specificity for FSCs and their immediate progeny (Figure 4A) (Hartman et al., 2010). This robust and useful expression pattern suggested that the associated gene likely was expressed and possibly functional in FSCs. Using the Splinkerette PCR method (Potter \& Luo, 2010), a 500bp band of genomic DNA was isolated from 109-30-Gal4 flies, matching the insertion locus (Figure 4B). Sequencing revealed that 109$30-G a l 4$ is inserted in the sickie (sick) gene, a known regulator of axon growth in mammals, worms, and flies (Abe et al., 2014; Coy et al., 2002; Maes, Barceló, \& Buesa, 2002; Merrill, Plum, Kaiser, \& Clagett-Dame, 2002; Schmidt et al., 2009). A second Gal4 insertion, sick ${ }^{\text {MIO8398-TG4.0, }}$ revealed the same pattern of expression in FSCs (Figure 4C), and the lethal allele, sick ${ }^{N P 0608}$, failed to complement 109-30 Gal4, confirming the identity of 109-30 Gal4 as sick-Gal4. Strikingly, sickie signals downstream of still life to control axonal outgrowth (Figure 4D) (Julian Ng \& Liqun Luo, 2004; Zheng et al., 2016), suggesting an important role of this pathway in FSC projection extension. Similar to the effects of sif on FSC projection growth, sick FSC knockdown resulted in short, thickened projections (Figure 2E, 3C,D). Proliferation at 6 hours after feeding was not affected by reduced sick expression (Figure 3B), emphasizing that induction of proliferation and projection growth are separable during the quiescence to proliferation transition. Importantly, sick and sif were sufficient to drive projection growth in nutrient-restricted flies, with overexpression of either gene increasing projection length (Figure 4E-G). Proliferation was not induced under these conditions (Figure $4 \mathrm{H}$ ).

Defects in FSC projections affect germline cyst organization and cells of contact

Homotypic interactions between FSC projections create a barrier-like network in the plane of the FSC niche (Hartman et al., 2015). Germline cysts encounter this barrier upon transitioning away from contacts with the inner germarial sheath (IGS/escort) cells in the anterior of the germarium to become encapsulated by follicle cells and form
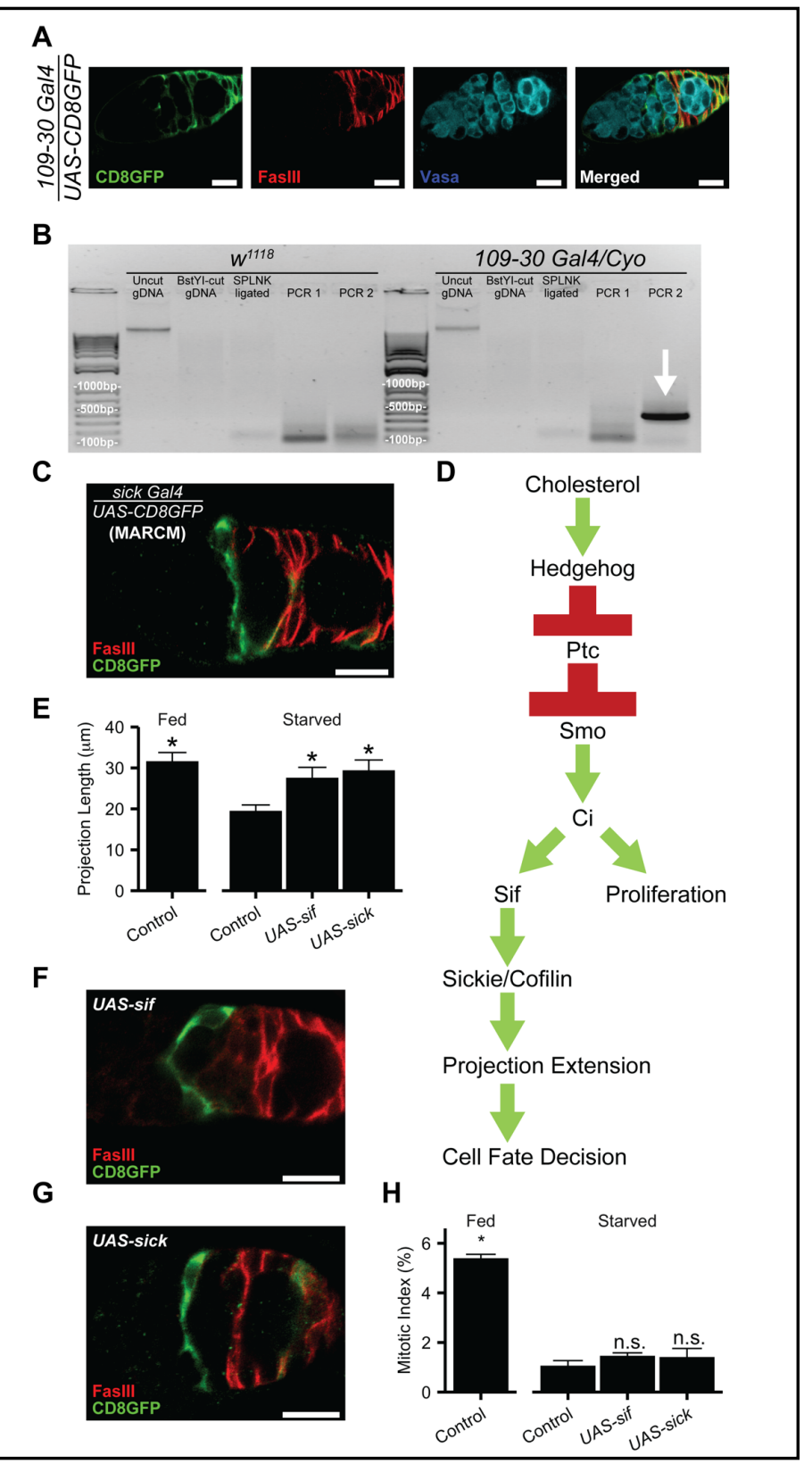

Figure 4: sickie and still life induce projection extension in nutrient restricted FSCs.

(A) Pattern of 109-30 Gal4 expression, indicated by UAS-mediated activation of CD8GFP (green) expression. Germ cells (Vasa, teal) and follicle cells (FasIII, red) are labeled. (B) Splinkerette PCR rescued $500 \mathrm{bp}$ fragment from $109-30$ Gal4 flies. Sequencing revealed insertion in the sickie locus. (C) sick ${ }^{M 108398-T G 4.0}$ drives CD8GFP (green) expression in the 109-30 Gal4 pattern. Faslll (red) marks follicle cells. (D) Signaling model for sif- and sickie-mediated projection extension regulation. (E) Projection length in nutrientrestricted (starved) flies expressing sif or sickie transgenes under 109-30 Gal4 control. * $p<0.01$ when compared to control nutrientrestricted (starved). ${ }^{*} p<0.01$ between control fed and control starved. $\mathrm{N}>10$. (F, G) FSCs expressing CD8GFP (green) under 109-30 Gal4 control in nutrient-restricted FSCs also overexpressing sif $(F)$ or sickie (G). (H) FSC proliferation upon overexpression of sif WT and sickie WT in nutrient-restricted flies shown as mitotic index (germaria with $\mathrm{PH} 3+\mathrm{FSC} /$ total). ${ }^{*} p<0.01$ or n.s. (no statistical significance) when compared to control nutrient-restricted (starved). (A,C,F) Scale bars are $10 \mu \mathrm{m}$. 
egg chambers (Figure 1A). Control FSCs create a barrier network that spans the germarium, interacting with flattened germline cysts during the transition period (Figure 5A) (Hartman et al., 2015). Barrier formation is dramatically altered upon sick or sif knockdown, only partially spanning the germarium and extending outside the plane of the FSC niche (Figure 5B). This results in disrupted germline cyst architecture, with cysts moving into the niche side-byside or with grossly abnormal organization (Figure $5 B, C)$. Aberrant organization during the encapsulation process has developmental implications, with egg chamber defects including multiple cysts packaged into one egg chamber and disruption of individual egg chambers (Figure 5C,D), demonstrating a functional role of projections in egg chamber formation.

sick knockdown FSC projections shift their cellular targets relative to control FSCs. Whereas control FSCs predominantly form homotypic, FSC-FSC contacts (57\%, Figure 2B,E), sick knockdown FSC projections frequently stall $(49-50 \%)$, reducing FSCFSC interactions (21-23\%) without dramatically affecting FSC-GC contact (Figure 2D,E). This suggests that projection length is important for determining the cells of contact, with homotypic, FSC-FSC interactions requiring substantial projection growth.

FSC projections regulate the self-renewaldifferentiation equilibrium

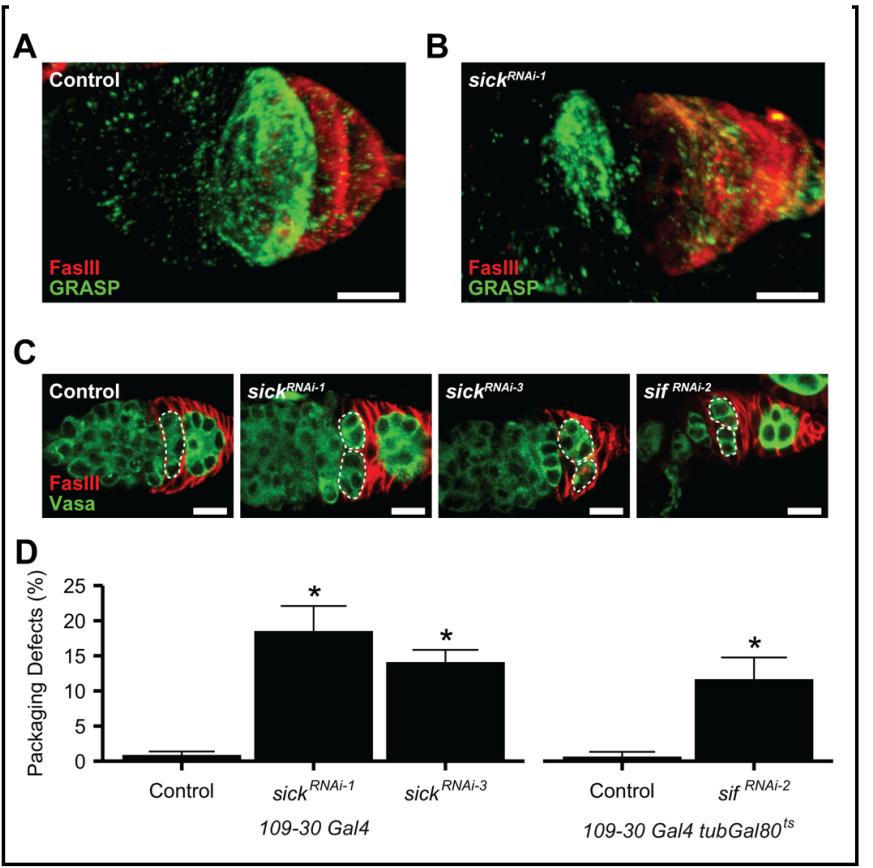

Figure 5: Loss of the FSC projection barrier network affects egg chamber formation.

$(\mathrm{A}, \mathrm{B})$ 3-dimensional image of the FSC projection barrier network

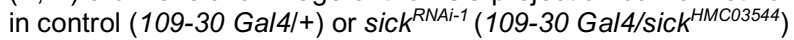
germaria. FasIll (red) marks follicle cells. GRASP (green) marks FSCs and projections. (C) Germline cysts (Vasa, green, white dotted outline) flatten across the germarium and are packaged as single units by follicle cells (red). Reduced sick or sif expression leads to aberrant packaging, with side-by-side or broken cysts. (D) Quantification of packaging defects: germaria with defect/total. * $p<0.01$ when compared with $109-30$ Gal4 or 109-30 tub Gal80 ts controls (indicated). N>129. (B,C) Scale bars are $10 \mu \mathrm{m}$.

process (Chang, Jang, Lin, \& Montell, 2013; W. Dai et al., 2017). Both proteins are expressed at equal, but low levels at the Region 2A/2B border in the plane of the FSC niche. Expression remains equal as cells enter Region 2B, with levels increasing over that observed at the Region 2A/2B border. In Region 3, where germline cysts are fully encapsulated by follicle cells, Eya and Cas expression dictates cell fate. Increased Cas and decreased Eya specify polar and stalk cells, whereas increased Eya and decreased Cas specify the main body follicle cells that surround germline cysts (W. Dai et al., 2017). We found that Eya protein expression is feeding dependent (Figure 6A), with low levels observed in FSCs in nutrient-restricted conditions and a dramatic increase within 24 hours of feeding. Cas levels remained similar in nutrient-restricted versus fed flies (Figure 6A), suggesting Eya is specifically affected by feeding. This is consistent with prior work demonstrating that Eya expression is regulated by the $\mathrm{Hh}$ effector patched (ptc), with loss of ptc (increased Hh signaling) associated with increased Eya expression (W. Dai et al., 2017).

The relative ratios of Eya and Cas instruct fate decisions (Chang et al., 2013; W. Dai et al., 2017), providing an opportunity to evaluate the impact on the differentiation process of altered FSC projection function upon sick knockdown. FSCs reside at the border between germarium region 2A and 2B (Figure 1A) (Margolis \& Spradling, 1995; Nystul \& Spradling, 2007; Reilein et al., 2017). The precise location is the subject of some debate, based on differential interpretation of lineage tracing studies (Fadiga \& Nystul, 2019; Nystul \& Spradling, 2007; Reilein et al., 2017). Part of the challenge in interpretation may 
be technical, with short-term proliferation activity among a subset of cells resident within the niche impacting lineage labeling. In addition, dynamic plasticity enables switching of cellular location within the niche to alter the probability of self-renewal versus differentiation, with more posterior cells (Layer 1) likely to differentiate into follicle cells, anterior cells likely to generate IGS/escort cells (Layer 3), and central cells (Layer 2) having increased ability to self-renew (Chang et al., 2013; W. Dai et al., 2017; Reilein et al., 2017; Reilein et al., 2018; C. Vied, Reilein, Field, \& Kalderon, 2012). The concept that stem cell lineage survival is determined by position is also conserved in mammals (Corominas-Murtra et al., 2020). To evaluate the impact of FSC projections on the self-renewal versus differentiation cell fate decision, we quantified Eya and Cas expression in three Layers of cells centered at the Region 2A/2B border (Figure 1A) (W. Dai et al., 2017). Consistent with published work, we found increasing expression of Eya and Cas from anterior to posterior (Figure 6C). Eya and Cas expression increased in Region 2B/Layer 1 cells, located immediately adjacent to cells expressing high levels of the polarization marker Fas III (Figure 6B,C). Finally, cells in Region 2A/Layer 3 exhibited low to undetectable levels of Eya and Cas, reflecting their identity as posterior IGS/escort cells (Figure 6B,C). sick and sif knockdown FSCs at the Region 2A/2B border/Layer 2 exhibited dramatically increased expression of Cas (Figure 6C). sick knockdown also resulted in significant upregulation of Eya in the same cells (Figure 6C). No differences relative to controls were observed in Region 2B/Layer 1 or Region 2A/Layer 3 cells, strongly indicating that the defects observed arise due to sickie and still life function in FSCs at the Region 2A/2B border.

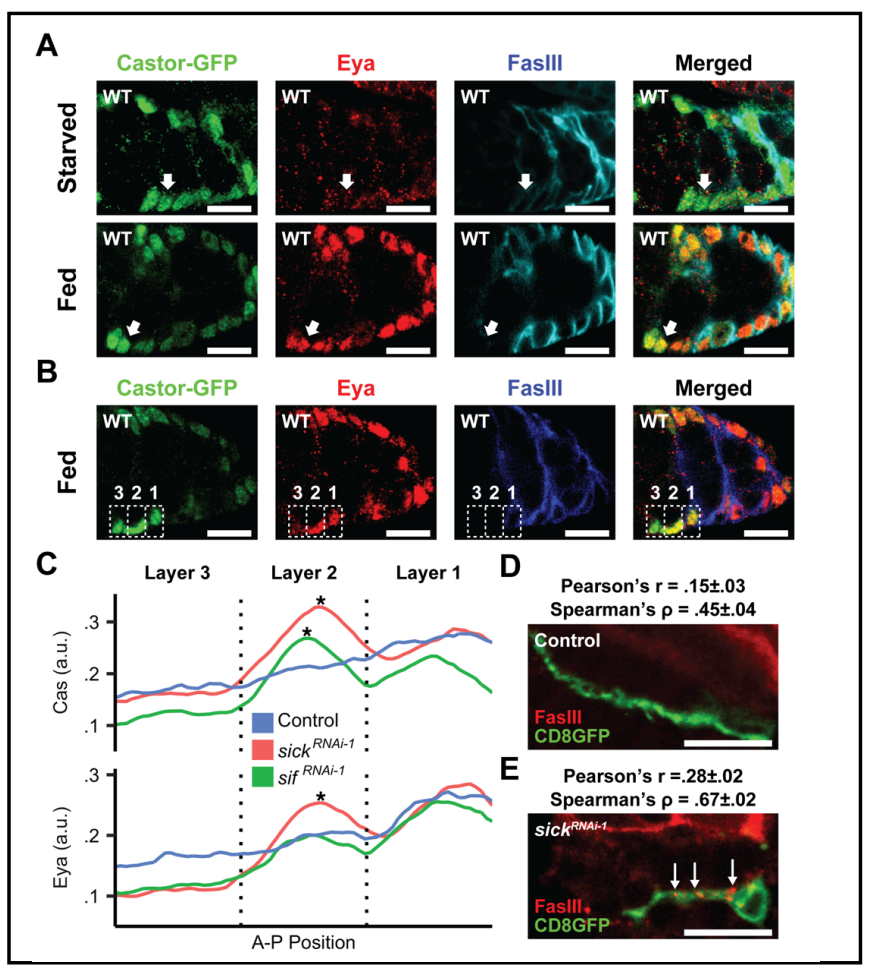

Figure 6: FSCs differentiate when sif or sickie expression is reduced.

$(\mathrm{A}, \mathrm{B})$ Eya (red) and Cas (green) expression in nutrient-restricted (starved) versus 24 hour fed (fed) flies. Follicle cells (blue) and merged images are shown. Cas expression is constitutive in FSCs at the Region 2A/2B border/Layer 2 (white arrow), whereas Eya is reduced in nutrient-restricted FSCs but robustly expressed upon feeding. Layers 1,2,3 are indicated in (B). (C) Average fluorescence intensity of $>182$ FSC niche images of Castor (top), Eya (bottom) (Wei Dai, Amy Peterson, Thomas Kenney, Haley Burrous, \& Denise J. Montell, 2017). *BHcorrected $p<1 \mathrm{e}-4$ when compared with indicated Layer 2 control. (a.u.) = arbitrary unit. $(D, E)$. Differentiation marker expression in FSCs and projections in fed flies. Co-localization of CD8GFPlabeled FSC projections (green) and FasIII (red), indicated by white arrows, increases significantly in sick ${ }^{R N A i}$ mutants.

Pearson's and Spearman's correlation coefficients range from -1 to 1.1 indicates complete colocalization, 0 indicates an absence of correlation. Both metrics show an increase in colocalization in sick mutant projections relative to control. (A,B,D,E) Scale bars

After egg chambers form, low Eya expression and high Cas expression drives the polar and stalk cell fate. This cell fate decision is characterized by dramatic upregulation of the polarity protein Fas3, which is a definitive marker of polar and stalk cells throughout oogenesis(Bai \& Montell, 2002; Ruohola et al., 1991). Strikingly, sick knockdown FSCs exhibited aberrant upregulation of Fas3, with strong puncta of Fas3 staining along FSC projections (Figure $6 \mathrm{D}, \mathrm{E}$ ). These results support the prevailing notion that the Eya-Cas equivalency maintains FSC plasticity, and disruption of the balance promotes induction of differentiation markers such as Fas3 (Chang et al., 2013; W. Dai et al., 2017). 


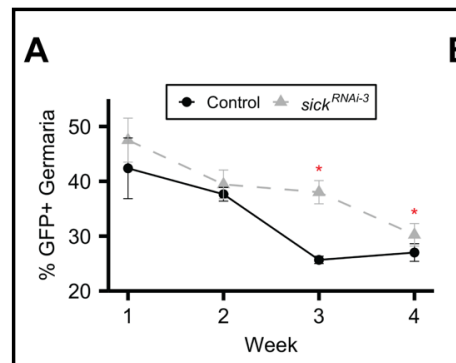

B
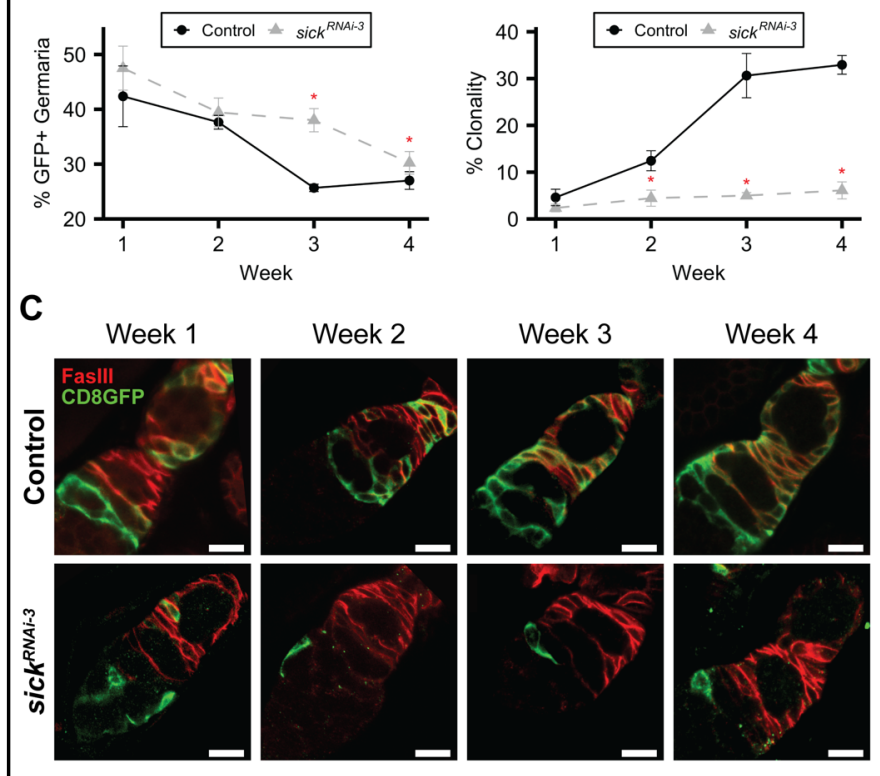

Figure 7: Age-dependent loss of FSC function in sick knockdown FSCs.

(A) Percent of germaria bearing control or sick ${ }^{R N A i}$ expressing GFPmarked FSCs over a 4-week timecourse. ${ }^{*} p<0.01$ ( $\chi^{2}$ test) when compared to control (109-30 Gal4/CD8GFP) (B) Percent of fully clonal germaria, with all FSC progeny labeled by GFP. ${ }^{*} p<0.01\left(\chi^{2}\right.$ test) when compared to control (109-30 Gal4/CD8GFP). N>367.

(C) Representative images of the 4-week timecourse showing CD8GFP-labeled FSCs (green) and follicle cells (red). Control FSCs generate CD8GFP labeled follicle cells, increasing with age. In contrast, sick ${ }^{R N A i}$ mutant FSCs lose functionality, with no follicle cell progeny by 3 weeks after feeding. Scale bars are $10 \mu \mathrm{m}$.
The sick knockdown FSCs were unusual, however. Whereas most FSCs undergoing differentiation leave the niche and become incorporated into the follicular epithelium (Nystul \& Spradling, 2007; Smith, Cummings, \& Cronmiller, 2002; Song \& Xie, 2002; Ulmschneider et al., 2016; Zhang \& Kalderon, 2000), reducing sick in FSCs led to higher retention rates than control three and four weeks after feeding (Figure 7A). Although sick knockdown did not impact FSC proliferation during the quiescence to proliferation transition in young flies (Figure 2), sick knockdown FSCs lost function rapidly during aging, as no clonally labeled daughter cells were produced after week 3 (Figure 7C). Whereas labeled control FSCs often dominate the niche to generate completely clonal follicular epithelia over time, sick knockdown FSCs rarely were able to compete (Figure 7B,C). These results suggest that defective projections impact FSC functionality, with precocious differentiation linked with extended occupancy of limited niche space, resulting in diminished ability to produce progeny over time.

\section{Discussion:}

FSCs have long been studied for their role in generation of the follicular epithelium that ensures proper oocyte development (Margolis \& Spradling, 1995). Substantial work has identified signals that promote FSC proliferation, self-renewal, and long-term retention in the niche, utilizing this system as a model for understanding how stem cells residing in a competitive niche environment retain the ability to continuously produce cells that maintain a healthy, functional tissue over a lifetime. Here, we show that axon-like projections are extended by FSCs in response to feeding in a manner that depends on the axon-growth regulators still life and sickie. Projections mediate dynamic FSC-FSC or FSC-germline interactions, with different consequences depending on the target cell. Our results are consistent with a model in which FSC projections transmit signals that determine FSC fate, with FSC-FSC interactions promoting self-renewal, and their disruption permitting differentiation as a default state.

In addition to homotypic contact, FSC projections interact heterotypically with germline cysts. Previous work demonstrated that contact between follicle cells and germline cysts induces polarization of the epithelial cells (Bhat et al., 1999; Bilder et al., 2000; Goode et al., 1996; Tanentzapf et al., 2000), an early step in induction of differentiation. Our results suggest that this induction mechanism may be indirect $(\mathrm{Li}$, Han, \& Xi, 2010). Whereas heterotypic interactions between FSC projections and germ cells are similar between control and sick knockdown FSCs (Figure 2), sick knockdown results in upregulation of 
differentiation markers, including the polarity marker Fas3 (Figure 6). If germ cell contact was the only mechanism driving differentiation, then FSC projections with reduced sick should exhibit increased heterotypic FSC-GC interactions to explain the enhanced differentiation phenotype. Instead, projections in sick knockdown conditions are frequently stalled (Figure 3), a defect that correlates with upregulation of differentiation markers in the absence of germ cell interaction. This suggests that disruption of FSCFSC homotypic interactions, either due to stalled projection growth or breakage during passage of germline cysts through the FSC niche, may drive differentiation, rather than direct induction upon germ cell contact.

The idea that FSC-FSC interactions are determinative for FSC fate determination raises questions about the mechanism. FSC projections clearly are major contributors to the architecture of the germarium, forming a web-like network that spans the niche (Figure 5). Projections with reduced sick expression are able to interact with both FSCs and germline cysts, but morphological defects and reduced length result in aberrant web formation that disrupts egg chamber formation. Whereas germline cysts in controls flatten across the germarium at the location of the projection web, they remain more spherical upon sick knockdown, often crossing the FSC niche side-by-side (Figure 5). Mispackaged egg chambers are prevalent, often with more than one germline cyst packaged into a single follicle (Figure 5). These results suggest that FSC projection networks are critical for proper egg chamber formation. sick knockdown FSC projections also may lose the ability to recognize germline cysts as a unit, given their unexpected ability to break germ cells off the cyst during the encapsulation process (Figure 5C).

We identified the well-known axon growth regulators still life and sickie as key regulators of FSC projection growth and organization in response to feeding. The Sif-Sick pathway controls the activity of Cofilin, an actin severing protein (Abe et al., 2014; Coy et al., 2002; Maes et al., 2002; Merrill et al., 2002; Julian Ng \& Liqun Luo, 2004; Schmidt et al., 2009). Actin dynamics in the form of polymerization (mediated by Cdc42/Arp2) and depolymerization (mediated by Cofilin) are essential for axonal growth (Dent, Gupton, \& Gertler, 2011; Flynn et al., 2012; Hall \& Lalli, 2010; J. Ng \& L. Luo, 2004; Julian Ng \& Liqun Luo, 2004). The actin cytoskeleton creates protrusions that are entered by microtubules, which initiate extension from the cell body and drive axonal growth in a Cofilin-dependent manner (Flynn et al., 2012; Gallo, 2011; Sudarsanam, Yaniv, Meltzer, \& Schuldiner, 2020). Actin dynamics also are necessary along the length of the axon, creating branches that form communication contacts with target cells and sense the environment during outgrowth (Dent et al., 2011). Striking similarities exist between FSC projections and axons, including dependence on Sif-Sickie-Cofilin-mediated actin depolymerization and Cdc42-Arp2-mediated polymerization (this work), as well as similar growth dynamics and sending of fine extensions along the FSC projection length to sense or interact with target cells (Figure 2) (Gallo, 2011).

The observation that expression of sif or sick in starved FSCs drives FSC outgrowth (Figure 4) emphasizes that this pathway is both necessary and sufficient for controlling this process. In fed flies, activation of Smo by $\mathrm{Hh}$ may lead to recruitment and activation of Sif, similar to activation of the Sif homolog, TIAM-1, in mammalian cells (Sasaki et al., 2010). Alternatively, Ci may activate expression of genes needed for sif or sick function. Expression of sif and sick is independent of both feeding and $\mathrm{Ci}$ activity in FSCs (data not shown), making it unlikely that they are direct Ci targets. In contrast, regulators of Cofilin activity, including Protein Kinase D, are upregulated in response to feeding (data not shown), suggesting that Hh transcriptional targets may be needed to establish dynamic on-off states for Cofilin activity. Coordination of Smo-dependent roles and transcriptional responses likely drives initial projection outgrowth, with long-term dynamics in fed states relying on sustained activation of both mechanisms.

In addition to shared use of cytoskeletal regulatory mechanisms for outgrowth, we previously showed that FSC projections resemble axons, with a microtubule core and dependence on integrin-mediated regulation for directed growth (Gallo, 2011; Hartman et al., 2015; Sudarsanam et al., 2020) (Figure 2). Cytoplasmic projections that transmit stem cell regulatory signals have been identified in other systems as well. Most prominently, thin, specialized filopodia called cytonemes extend transiently from niche cells 
to deliver signals, including $\mathrm{Hh}$, to regulate stem cell self-renewal and function (Casas-Tintó \& Portela, 2019; Fuwa, Kinoshita, Nishida, \& Nishihara, 2015; González-Méndez, Gradilla, \& Guerrero, 2019; Inaba, Buszczak, \& Yamashita, 2015; Junyent et al., 2020; Rojas-Ríos, Guerrero, \& González-Reyes, 2012; Snyder et al., 2015). FSCs are the first documented non-neuronal case where $\sim 40 \mu \mathrm{m}$ long, microtubulecontaining, projections that are stable to fixation provide both structural information and communication within the stem cell niche. FSCs exhibit inherent asymmetry, with a long, primary projection and a shorter secondary projection (Figure 2B), reminiscent of axon-dendrite organization in developing neurons. Importantly, FSC projections transmit information. Levels of Eya and Cas in FSCs depend on proper projection length, with stalled and misdirected projections leading to a shift from a self-renewing, stem cell signature to a state reminiscent of polar or stalk cell differentiation. Most likely, signals transmitted between FSCs activate pathways that prevent upregulation of Eya and Cas, to maintain a plastic, selfrenewing state. An appealing model is that primary and secondary FSC projections interact, forming an axon-dendrite-like communication conduit that transmits signals needed to suppress differentiation and maintain self-renewal. This would explain how stalled FSC projections impact cell fate, as the shortened "axons" would fail to contact target "dendrites" due to failure to reach. Identification of key signals transmitted between FSCs will shed light on a longstanding mystery of how stem cell fate is determined.

An interesting distinction between axons and FSC projections is their permanency. Whereas axons generally identify a cellular target and remain associated throughout the lifespan in the absence of injury, FSCs projections are highly dynamic in a feeding-dependent manner (Figure 1). This may contribute to equalization of FSCs within the niche during periods of quiescence when projections are short and development arrests. This method of reducing competitive advantage is proliferation-independent (Figures 1,3), perhaps indicating that re-establishment of homotypic connections by select FSCs after breakage of the projection barrier by germline cysts is advantageous relative to FSC-germline connections for long-term niche retention. Surprisingly, we found that sick knockdown FSCs were retained at the same rate, or longer than controls (Figure 7), supporting a model in which activation of differentiation pathways via cell polarization may enhance adhesion to the niche. Over time, filling the niche with precociously differentiated FSCs is expected to contribute to functional decay of the stem cell pool. The direct regulation of both FSC proliferation and projection outgrowth by $\mathrm{Hh}$ emphasizes its central role in long term stem cell function and provides opportunities for uncovering mechanisms that might be exploited for regenerative medicine.

\section{Acknowledgments:}

We thank J.R. Peterson, D. Ruiz-Whalen, and N. Fried for insight and comments, A. Mount and V. Chan for imaging help, and R. Lehmann for anti-Zfh1 antibodies. We also thank Drosophila resource centers at Bloomington [NIH P40OD018537], Vienna (VDRC, www.vdrc.at), and Kyoto (DGRC, Kyoto Institute of Technology), and the Developmental Studies Hybridoma bank (NICHD and University of lowa). This work was supported by NIH [R01 HD065800] (AOR), T32 CA009035 (E. Lee), and P30 CA06927 (FCCC)], the Bucks County Board of Associates, and a Grateful Kidney Cancer Patient fund at FCCC (J. Reinach).

\section{Author contributions:}

Conceptualization: E.L. and A.OR, Methodology: E.L, A.OR, D.Z., Validation, Formal Analysis, Data Curation: D.Z., E.L., A.J., Investigation: E.L., A.OR., M.W., J.R., K.C., C.C., A.V., J.A. Writing-Original Draft: A.OR, E.L., Writing-Review and Editing: E.L. A.OR, D.Z., J.A., Visualization: D.Z., E.L., Supervision: A.OR, Project Administration, Funding Acquisition: A.OR, E.L.

\section{Declaration of Interests:}

The authors declare no competing interests. 
bioRxiv preprint doi: https://doi.org/10.1101/2020.11.07.372664; this version posted November 8, 2020. The copyright holder for this preprint (which was not certified by peer review) is the author/funder, who has granted bioRxiv a license to display the preprint in perpetuity. It is made available under aCC-BY-NC 4.0 International license. 


\section{Figure Legends:}

Figure 1. Hedgehog signaling regulates FSC proliferation and projection length.

(A) Schematic diagram of the germarium. FSCs (green) are located at the Region 2A/2B border, also called Layer 2. Cells in Region 2B/Layer1 (yellow, also called pre-follicle cells) preferentially produce follicle cells (red), but retain the capacity to self-renew or change position with the FSC niche. Cells in Region 2A/Layer 3 (blue), adopt an escort cell fate, but self-renew and generate follicle cells on rare occasions. Germline cysts (peach), interact with FSCs and become encapsulated by follicle cells to form egg chambers. Apical cells (gray rectangles), Germline Stem Cells (gray circles), Cystoblast (gray oval) and Inner Germarial Sheath (IGS/escort cells, gray triangles) are also shown in Region 1. (B) Representative image of FSC primary and secondary projections. Top, FSC (green), and follicle cells (red). Bottom, FSC only. Faslll (red) marks follicle cells. CD8GFP (green) marks FSCs and projections. (C) Time course of proliferation and projection extension. Flies were nutrient restricted (starved) prior to feeding of yeast $0,3,6,12$, and 24 hour timepoints after feeding are indicated. Frequency of germaria with at least one FSC in mitosis $(\mathrm{PH} 3+)$ is shown. Projection length $(\mu \mathrm{m})$ was measured in MARCM GFP labeled FSCs at the indicated timepoints. (D) Quantification of proliferation frequency as measured by mitotic index (germaria containing PH3+ FSC/total), indicated for each genotype. Flies were nutrient-restricted (starved) for 3 days or fed for 6 hours after a 3 day nutrient restriction (fed). $\mathrm{N}>322$. (E) Projection length $(\mu \mathrm{m})$ of MARCM GFP labeled primary projections in flies nutrientrestricted (starved) for 3 days or fed 6 hours after 3 days of nutrient restriction (fed). $N>6$. ( $F$ ) Representative FSC projection images. CD8GFP (green) marks FSCs and projections. FasllI (red) marks follicle cells. ${ }^{*} p<0.01$ when compared to indicated control. $(B, F)$ Scale bars are $10 \mu \mathrm{m}$.

Figure 2: FSC projections exhibit homotypic and heterotypic interactions.

(A) Schematic of CoinFLP. After heat shock-induced mitotic recombination, one cell expresses exon 11 of split GFP fused to the transmembrane protein CD4 (CD4-spGFP11) under Actin-LexA control and a neighboring cell expresses exons 1-10 of split GFP fused to CD4 under Actin-Gal4 control.

Transcellular binding reconstitutes the GFP, producing green fluorescence (GRASP). (B) Homotypic FSC-FSC interactions. FasIII (red) marks follicle cells. GRASP (green) marks FSCs and projections. C) Heterotypic interactions between FSC projections and germline cyst. (D) sick ${ }^{\text {RNAi }}$ FSCs exhibit stalled projections. (E) Quantification of FSC projection interactions. N>183. (B-D) Vasa (blue) marks germ cells. FasllI (red) marks follicle cells. GRASP (green) marks FSCs and projections. Scale bars are 10 $\mu \mathrm{m}$.

Figure 3: Candidate screen for FSC projection regulators.

(A) FSC proliferation in control versus dominant negative or RNAi knockdown mutants, scored as mitotic index (germaria with PH3+ FSC/total). UAS-Cdc42 ${ }^{L 89}$ and $A r p 2^{J F 02585}$ values are from continuously fed flies. sif ${ }^{R N A-1 i}$ values are from flies nutrient restricted for 3 days and then fed 6 hours. ${ }^{*} \mathrm{p}<0.01$ when compared to 109-30 tub Gal80 $/+$. N $>357$. (B) FSC proliferation in sick ${ }^{R N A i}$ mutants 6 hours after feeding nutrient restricted flies. $N>509$. (C) Primary projection length in indicated genotypes. ${ }^{*} p<0.01$ when compared to 109-30 Gal4/+. N>6. (D) Representative images of projections. CD8GFP (green) marks FSCs and projections. FasIll (red) marks follicle cells.

Figure 4: sickie and still life induce projection extension in nutrient restricted FSCs.

(A) Pattern of 109-30 Gal4 expression, indicated by UAS-mediated activation of CD8GFP (green) expression. Germ cells (Vasa, teal) and follicle cells (FasllI, red) are labeled. (B) Splinkerette PCR rescued 500bp fragment from 109-30 Gal4 flies. Sequencing revealed insertion in the sickie locus. (C) sick $^{\text {MI08398-TG4.0 }}$ drives CD8GFP (green) expression in the 109-30 Gal4 pattern. FasIII (red) marks follicle cells. (D) Signaling model for sif- and sickie-mediated projection extension regulation. (E) Projection length in nutrient-restricted (starved) flies expressing sif or sickie transgenes under 109-30 Gal4 control. ${ }^{*} p<0.01$ when compared to control nutrient-restricted (starved). ${ }^{*} p<0.01$ between control fed 
and control starved. N>10. (F, G) FSCs expressing CD8GFP (green) under 109-30 Gal4 control in nutrient-restricted FSCs also overexpressing sif $(\mathrm{F})$ or sickie $(\mathrm{G})$. $(\mathrm{H}) \mathrm{FSC}$ proliferation upon overexpression of sif WT and sickie WT in nutrient-restricted flies shown as mitotic index (germaria with $\mathrm{PH} 3+\mathrm{FSC} /$ total). ${ }^{*} p<0.01$ or n.s. (no statistical significance) when compared to control nutrientrestricted (starved). (A,C,F) Scale bars are $10 \mu \mathrm{m}$.

Figure 5: Loss of the FSC projection barrier network affects egg chamber formation. (A, B) 3-dimensional image of the FSC projection barrier network in control (109-30 Gal4/+) or sick RNAi-1 $^{\text {G }}$ (109-30 Gal4/sick ${ }^{H M C 03544}$ ) germaria. FasIll (red) marks follicle cells. GRASP (green) marks FSCs and projections. (C) Germline cysts (Vasa, green, white dotted outline) flatten across the germarium and are packaged as single units by follicle cells (red). Reduced sick or sif expression leads to aberrant packaging, with side-by-side or broken cysts. (D) Quantification of packaging defects: germaria with defect/total. * $p<0.01$ when compared with $109-30 \mathrm{Gal}$ or $109-30$ tub Gal80 ${ }^{\text {ts }}$ controls (indicated). $\mathrm{N}>129$. $(\mathrm{B}, \mathrm{C})$ Scale bars are $10 \mu \mathrm{m}$.

Figure 6: FSCs differentiate when sif or sickie expression is reduced.

$(A, B)$ Eya (red) and Cas (green) expression in nutrient-restricted (starved) versus 24 hour fed (fed) flies. Follicle cells (blue) and merged images are shown. Cas expression is constitutive in FSCs at the Region 2A/2B border/Layer 2 (white arrow), whereas Eya is reduced in nutrient-restricted FSCs but robustly expressed upon feeding. Layers 1,2,3 are indicated in (B). (C) Average fluorescence intensity of $>182$ FSC niche images of Castor (top), Eya (bottom)(Wei Dai et al., 2017). *BH-corrected $p<1 e-4$ when compared with indicated Layer 2 control. (a.u.) = arbitrary unit. (D,E). Differentiation marker expression in FSCs and projections in fed flies. Co-localization of CD8GFP-labeled FSC projections (green) and FasIII (red), indicated by white arrows, increases significantly in sick ${ }^{R N A i}$ mutants. Pearson's and Spearman's correlation coefficients range from -1 to 1.1 indicates complete colocalization, 0 indicates an absence of correlation. Both metrics show an increase in colocalization in sick mutant projections relative to control. $(A, B, D, E)$ Scale bars are $10 \mu \mathrm{m}$.

Figure 7: Age-dependent loss of FSC function in sick knockdown FSCs.

(A) Percent of germaria bearing control or sick ${ }^{R N A i}$ expressing GFP-marked FSCs over a 4-week timecourse. ${ }^{*} p<0.01$ ( $\chi^{2}$ test) when compared to control (109-30 Gal4/CD8GFP) (B) Percent of fully clonal germaria, with all FSC progeny labeled by GFP. ${ }^{*} p<0.01\left(\chi^{2}\right.$ test $)$ when compared to control (109-30 Gal4/CD8GFP). N>367. (C) Representative images of the 4-week timecourse showing CD8GFP-labeled FSCs (green) and follicle cells (red). Control FSCs generate CD8GFP labeled follicle cells, increasing with age. In contrast, sick ${ }^{R N A i}$ mutant FSCs lose functionality, with no follicle cell progeny by 3 weeks after feeding. Scale bars are $10 \mu \mathrm{m}$. 


\section{Materials and Methods:}

\section{Fly Preparation}

All fly stocks were raised on standard food available from the in-house facility at Fox Chase Cancer Center. Flies were maintained at standard $25^{\circ} \mathrm{C}$, additional fly stocks were maintained at $18^{\circ} \mathrm{C}$ temperature-controlled incubators.

\section{Fly Strains and genetics}

The following stocks were obtained from the Bloomington Drosophila Stock Center (BDSC, Bloomington,

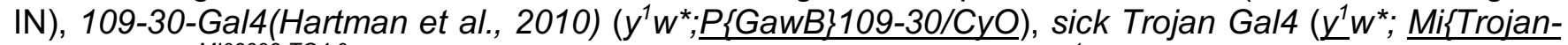
GAL4.0\}sick ${ }^{\text {MIO8398-TG4.0 }}$ SM6a), smo RNAi (Hartman et al., 2010) ( $y^{1} w^{*} ; P\{w[+m C]=U A S-s m o . R N A i\} 2$ P\{UAS-smo.RNAi\}8/CyO, P\{Wee-P.ph0\}2), Ci RNAi (Singh, Lee, Hartman, Ruiz-Whalen, \& O'Reilly, 2018) ( $y v$; P\{TRiP.JF01715\}attP2), UAS-string (Singh et al., 2018) ( $w^{1118}$; P\{UAS-stg.N\}4), cdc42 dominant negative ( $\left.w^{*} ; P\{U A S-C d c 42 . L 89\} 4\right)$, arp2 RNAi $\left(y^{1} v^{1} ; P\{T R i P . J F 02785\} a t t P 2 / T M 3, S^{1}\right)$, sif RNAi-1 ( $y^{1} v^{1} ; P\left\{\right.$ TRiP.JF01795\}attP2), sif RNAi-2 ( $y^{1} v^{1} ;$ P\{TRiP.HMJ23517\}attP40), sick RNAi-1 ( $y^{1} v^{1}$; $P\{y[+t 7.7] \quad v[+t 1.8]=T R i P . H M J 21480\} a t t P 40), \quad$ sick $\quad R N A i-2 \quad\left(y^{1} v^{1} ; \quad P\{y[+t 7.7]\right.$ $v[+t 1.8]=T R i P . H M J 21863\} a t t P 40)$, sick RNAi-3 ( $\left.\underline{y}^{1} s{ }^{*} v^{1} \operatorname{sev}^{21} ; \underline{P}\{T R i P . H M C 03544\} a t t P 2\right), U A S-s i f\left(\underline{w^{*}} ;\right.$ $\underline{P}\{$ UAS-sif.S $\}$ M3.1). We have also obtained stocks from the Kyoto Stock Center (DGRC, Kyoto, Japan), UAS-sick ( $\left.y^{*} w^{*} ; P\{w+m C=U A S-s i c k . A\} 4844-1-8-M\right)$, sick-Gal4(w*;P\{GawB $\}$ sick $\left.^{* 00608} / C y O\right)$. Cas::GFP (FlyFos020486(pRedFlp-Hgr)(CG1211826169::2XTY1-SGFP-V5-preTEV-BLRP-3XFLAG)dFRT) was obtained from the Vienna Drosophila Resource Center (VDRC, Vienna, Austria). CoinFLP stocks (Bosch et al., 2015) were obtained from BDSC, $h s F L P\left(P\{r y[+t 7.2]=h s F L P\} 1, w^{1118} ; A d v / C y O\right)$, CoinFLP ( $w^{*}$; $P\{y[+t 7.7] w[+m C]=$ CoinFLP-LexA::GAD. GAL4\}attP40, $P\{w[+m C]=l e x A o p-r C D 2 . R F P\} 2 ; P\{w[+m C]=U A S-$ CD4-spGFP1-10\}3, $P\{w[+m C]=l e x A o p-C D 4-s p G F P 11\} 3 / T M 6 C, S b)$.

\section{Dissections, immunofluorescence, and microscopy}

Ovaries were dissected from adult flies in Grace's insect cell culture medium (Gibco, Gaithersburg, MD), fixed in 4\% paraformaldehyde for 15 minutes and then washed three times in 1X PBST for 5 minutes. The ovaries were then incubated with primary antibodies in $0.5 \%$ normal goat serum diluted with $1 \mathrm{X}$ PBST solution overnight at $4^{\circ} \mathrm{C}$. Primary antibodies used were mouse anti-Fasciclin III (Fas III) (1:200; DSHB, lowa City, IA; (Patel, Snow, \& Goodman, 1987)), mouse anti-Eya (1:40, DSHB (Boyle, Bonini, \& DiNardo, 1997)), rat anti-Vasa (1:10, DSHB (Aruna, Flores, \& Barbash, 2009)), rabbit anti-PH3 (1:1000, Millipore, Burlington, MA), rabbit anti-Zfh1 (1:1000, Gift from Ruth Lehmann), chicken anti-GFP (1:1000, Thermo Fisher Scientific, Waltham, MA). The ovaries were washed three times for 10 min each in 1X PBST and then incubated with secondary antibodies at RT for 1 hour. All secondary antibodies used were Alexa antibodies conjugated to species-specific secondary antibodies (1:200; Thermo Fisher Scientific). Ovaries were washed three times in 1XPBST. The ovaries were then mounted on slides using Vectashield medium (Vector Laboratories, Burlingame, CA).

\section{Creating Mosaic clones in germarium}

Mosaic analysis with repressible cell marker (MARCM) stocks were generated by crossing Ub-RFP, Gal80 FRT ${ }^{19 A}$ Flp ${ }^{122} / Y$; UAS-transgene males to FRT ${ }^{19 A}$; 109-30 Gal4/CyO females (Hartman et al., 2015). Flies were heat shocked for 1 hour at $37^{\circ} \mathrm{C}$ to obtain single clones of GFP positive labeled follicle stem cells. After the heat shock, female flies were kept at $25^{\circ} \mathrm{C}$ either in fly food vials or starved for on grape juice plates with males corresponding to different experimental design. Flies were kept in fresh vials for 3 days after heat shock before the ovaries were isolated. Germaria were stained with chicken anti-GFP and mouse anti-FasIll to image projections.

\section{Measurement of projection length}

After images of single cell GFP-labeled FSCs were acquired in the MARCM-labeled stocks, projections of germarium images were imported into IMARIS for measurement. Multi-point length measurements were taken from the center of the cell nucleus to the end of the projection by using the measurement 
function in IMARIS. Significant differences in projection lengths were determined using unpaired Student's $t$-tests using three biological replicates.

\section{FSC niche retention and clonality}

MARCM stocks were generated as described above. Flies were heat shocked at $37^{\circ} \mathrm{C}$ for 1 hour and placed in fresh vials subsequently at $25^{\circ} \mathrm{C}$. Flies were flipped into fresh vials twice a week to ensure food availability. Ovaries were dissected and stained with chicken anti-GFP and mouse anti-FasIII at week 1, 2,3 and 4 respectively. FSC niche retention was determined by scoring the percentage of germaria with GFP-positive clones in Region 2A/B. Functionality was determined by the presence of GFP-labeled FSC progeny in early stage egg chambers. Germaria with $100 \%$ of FSCs and follicle cells GFP-labeled were scored as fully clonal. Partial domination was not considered as clonal.

For hypothesis testing, the number of GFP-positive and GFP-negative germaria were summed across biological replicates for each genotype. For each week, a $\chi^{2}$ test of independence was performed to determine correlation between genotype and FSC retention. A $\boldsymbol{\chi}^{2}$ test of independence was also performed on GFP-positive germaria (fully clonal vs not clonal) to determine correlation between genotype and FSC clonality. $p$-values are reported with Yates correction.

\section{Proliferation Assay}

Flies were generated by crossing either 109-30 Gal4 Tub Gal80:CyO or 109-30 Gal4 to their corresponding UAS-transgene. Flies carrying 109-30 Gal4 TubGa/80 ${ }^{\text {ts }} / \mathrm{UAS}$-transgene were incubated at $29^{\circ} \mathrm{C}$ prior to dissection. All samples were starved for 3 days prior to feeding of yeast for 6 hours or one week. Ovaries were dissected in Grace's insect medium and stained with rabbit anti-phosphohistone-H3 $(\mathrm{PH} 3)$ and mouse anti-Faslll. After completing the immunofluorescence procedure described above, mitotic index was calculated as the number of germaria with at least one PH3-positive FSC, divided by the total number of germaria (Hartman et al., 2010; O'Reilly et al., 2008). Significant changes in mitotic index were determined by unpaired Student's $t$-test using three biological replicates.

\section{Packaging defects}

Flies were generated by crossing 109-30 Gal4 TubGal80/CyO or 109-30 Gal4 to their corresponding UAS-transgene. Flies carrying $109-30 \mathrm{Gal} 4 \mathrm{tubGa} / 80 / \mathrm{CyO}$ were incubated at $29^{\circ} \mathrm{C}$ prior to dissection. Ovaries were dissected in Grace's insect medium and stained with mouse anti-Faslll. Packaging defects were determined by observation of side-by-side germline cysts were in Region 3 (O'Reilly et al., 2008). Significant changes in incidence of packaging defects were determined by unpaired Student's $t$ tests using three biological replicates.

\section{CoinFlp Experiment}

CoinFlp (Bosch et al., 2015)experimental flies were generated by crossing $\mathrm{P}\{\mathrm{y}[+\mathrm{t} 7.7] \mathrm{w}[+\mathrm{mC}]=$ CoinFLPLexA::GAD.GAL4\}attP40,P $\{w[+m C]=l e x A o p-r C D 2 . R F P\} 2 ; \quad P\{w[+m C]=U A S-C D 4-s p G F P 1-10\} 3$, $\mathrm{P}\{\mathrm{w}[+\mathrm{mC}]=$ lexAop-CD4-spGFP11 $\} 3 / T M 6 C$ females to male flies with $h s F / p$; UAS-transgene. Flies were heat shocked for $1 \mathrm{~h}$ at $37^{\circ} \mathrm{C}$ and kept at $25^{\circ} \mathrm{C}$ in yeasted vials with males. After 3 days the ovaries were isolated and stained with chicken anti-GFP, mouse anti-FasllI, rat anti-Vasa for subsequent analysis.

\section{Quantification of Castor and Eya in FSCs}

Confocal images were processed using ImageJ. All images were taken in the cross section of the center of the germaria. Faslll expression was used to identify the germarium shape and FSC region. A region of interest (ROI) was determined by using Fas3 levels to establish three layers of FSCs in region 2A/2B, as described in Dai et al.(W. Dai et al., 2017) 
Signal intensity values from GFP (Cas) and Eya channels were extracted from each ROI, recorded along $X-Y$ coordinates, and imported into $R$ studio. Within each ROI, signal intensity values were normalized by dividing by maximum intensity within the ROI. All ROI coordinates were centered at the origin and truncated to a common ROI size (the minimum ROI width and height recorded across all samples).

For each channel, mean intensity was calculated at each $X-Y$ coordinate across replicates. For hypothesis testing, mean intensity values for sick and sif ROI were compared to control ROI for each channel, at each layer. $p$-values were determined from paired Student's $t$-tests on these values, with a Benjamini-Hochberg correction for multiple testing.

\section{Co-localization of FasIII and GFP expression}

Images of MARCM clones were analyzed by ImageJ. GFP-positive FSC projections were outlined as Region of Interest by polygon selection. The Coloc2 plug-in was used to analyze GFP and Faslll colocalization. Pearson's correlation coefficients (R) and Spearman's rank correlation coefficients $(\rho)$ were recorded and averaged between replicate images.

\section{Splinkerette PCR}

Splinkerette PCR (Potter \& Luo, 2010) was used to map the pGawB-GAL4 insertion in 109-30 Gal4 flies. Genomic DNA was isolated (Promega, Madison, WI) according to the manufacturer's protocol. Genomic DNA was digested by BstYI and ligated to Splinkerette oligonucleotides, followed by 2 rounds of PCR, exactly according to the published Splinkerette PCR protocol (Potter \& Luo, 2010). The 500bp DNA band was gel extracted (Qiagen, Germantown, MD) and sequenced.

\section{Image Analysis}

Images were collected at room temperature using 40X (1.25 NA) or 20X (0.7 NA) oil immersion lenses (Leica) on an upright microscope (DM 5000; Leica Microsystems, Wetzlar, Germany) coupled to a confocal laser scanner (TCS SP5; Leica). LAS AF SP5 software (Leica) was used for data acquisition. Images representing individual channels of single confocal slices or 3-dimensional reconstructions of the germarium, including the FSC region were exported into IMARIS or Fiji (ImageJ) for further analysis.

\section{Statistical Analysis}

Unless otherwise stated, statistical significance is reported as $p$-values generated from an unpaired Student's $t$-test. 


\section{References:}

Abe, T., Yamazaki, D., Murakami, S., Hiroi, M., Nitta, Y., Maeyama, Y., \& Tabata, T. (2014). The NAV2 homolog Sickie regulates F-actin-mediated axonal growth in Drosophila mushroom body neurons via the non-canonical Rac-Cofilin pathway. Development, 141(24), 4716-4728. doi:10.1242/dev.113308

Ables, E. T., Laws, K. M., \& Drummond-Barbosa, D. (2012). Control of adult stem cells in vivo by a dynamic physiological environment: diet-dependent systemic factors in Drosophila and beyond. Wiley Interdiscip Rev Dev Biol, 1(5), 657-674. doi:10.1002/wdev.48

Albert Hubbard, E. J., \& Schedl, T. (2019). Biology of the Caenorhabditis elegans Germline Stem Cell System. Genetics, 213(4), 1145. doi:10.1534/genetics.119.300238

Amoyel, M., Simons, B. D., \& Bach, E. A. (2014). Neutral competition of stem cells is skewed by proliferative changes downstream of $\mathrm{Hh}$ and Hpo. Embo J, 33(20), 2295-2313. doi:10.15252/embj.201387500

Aruna, S., Flores, H. A., \& Barbash, D. A. (2009). Reduced fertility of Drosophila melanogaster hybrid male rescue $(\mathrm{Hmr})$ mutant females is partially complemented by $\mathrm{Hmr}$ orthologs from sibling species. Genetics, 181(4), 1437-1450. doi:10.1534/genetics.108.100057

Bai, J., \& Montell, D. (2002). Eyes absent, a key repressor of polar cell fate during Drosophila oogenesis. Development, 129(23), 5377-5388. doi:10.1242/dev.00115

Bhat, M. A., Izaddoost, S., Lu, Y., Cho, K. O., Choi, K. W., \& Bellen, H. J. (1999). Discs Lost, a novel multiPDZ domain protein, establishes and maintains epithelial polarity. Cell, 96(6), 833-845. doi:10.1016/s0092-8674(00)80593-0

Bilder, D., Li, M., \& Perrimon, N. (2000). Cooperative regulation of cell polarity and growth by Drosophila tumor suppressors. Science, 289(5476), 113-116. doi:10.1126/science.289.5476.113

Bosch, J. A., Tran, N. H., \& Hariharan, I. K. (2015). CoinFLP: a system for efficient mosaic screening and for visualizing clonal boundaries in Drosophila. Development, 142(3), 597. doi:10.1242/dev.114603

Boyle, M., Bonini, N., \& DiNardo, S. (1997). Expression and function of clift in the development of somatic gonadal precursors within the Drosophila mesoderm. Development, 124(5), 971-982.

Bruens, L., Ellenbroek, S. I. J., Suijkerbuijk, S. J. E., Azkanaz, M., Hale, A. J., Toonen, P., . . van Rheenen, J. (2020). Calorie Restriction Increases the Number of Competing Stem Cells and Decreases Mutation Retention in the Intestine. Cell reports, 32(3), 107937-107937. doi:10.1016/j.celrep.2020.107937

Casas-Tintó, S., \& Portela, M. (2019). Cytonemes, Their Formation, Regulation, and Roles in Signaling and Communication in Tumorigenesis. International journal of molecular sciences, 20(22), 5641. doi:10.3390/ijms20225641

Chang, Y. C., Jang, A. C., Lin, C. H., \& Montell, D. J. (2013). Castor is required for Hedgehog-dependent cell-fate specification and follicle stem cell maintenance in Drosophila oogenesis. Proc Natl Acad Sci U S A, 110(19), E1734-1742. doi:10.1073/pnas.1300725110

Cho, I. J., Lui, P. P., Obajdin, J., Riccio, F., Stroukov, W., Willis, T. L., Watt, F. M. (2019). Mechanisms, Hallmarks, and Implications of Stem Cell Quiescence. Stem cell reports, 12(6), 1190-1200. doi:10.1016/j.stemcr.2019.05.012 
Çiçek, I. Ö., Karaca, S., Brankatschk, M., Eaton, S., Urlaub, H., \& Shcherbata, H. R. (2016). Hedgehog Signaling Strength Is Orchestrated by the mir-310 Cluster of MicroRNAs in Response to Diet. Genetics, 202(3), 1167-1183. doi:10.1534/genetics.115.185371

Clevers, H., \& Watt, F. M. (2018). Defining Adult Stem Cells by Function, not by Phenotype. Annual Review of Biochemistry, 87(1), 1015-1027. doi:10.1146/annurev-biochem-062917-012341

Corominas-Murtra, B., Scheele, C. L. G. J., Kishi, K., Ellenbroek, S. I. J., Simons, B. D., van Rheenen, J., \& Hannezo, E. (2020). Stem cell lineage survival as a noisy competition for niche access. Proceedings of the National Academy of Sciences, 117(29), 16969. doi:10.1073/pnas.1921205117

Coy, J. F., Wiemann, S., Bechmann, I., Bächner, D., Nitsch, R., Kretz, O., Poustka, A. (2002). Pore membrane and/or filament interacting like protein 1 (POMFIL1) is predominantly expressed in the nervous system and encodes different protein isoforms. Gene, 290(1-2), 73-94. doi:10.1016/s0378-1119(02)00567-x

Dai, W., Peterson, A., Kenney, T., Burrous, H., \& Montell, D. J. (2017). Quantitative microscopy of the Drosophila ovary shows multiple niche signals specify progenitor cell fate. Nature Communications, 8(1), 1244. doi:10.1038/s41467-017-01322-9

Dai, W., Peterson, A., Kenney, T., Burrous, H., \& Montell, D. J. (2017). Quantitative microscopy of the Drosophila ovary shows multiple niche signals specify progenitor cell fate. Nat Commun, 8(1), 1244. doi:10.1038/s41467-017-01322-9

de Navascués, J., Perdigoto, C. N., Bian, Y., Schneider, M. H., Bardin, A. J., Martínez-Arias, A., \& Simons, B. D. (2012). Drosophila midgut homeostasis involves neutral competition between symmetrically dividing intestinal stem cells. Embo J, 31(11), 2473-2485. doi:10.1038/emboj.2012.106

Demarco, R. S., Struckhoff, E. C., \& Lundquist, E. A. (2012). The Rac GTP Exchange Factor TIAM-1 Acts with CDC-42 and the Guidance Receptor UNC-40/DCC in Neuronal Protrusion and Axon Guidance. PLOS Genetics, 8(4), e1002665. doi:10.1371/journal.pgen.1002665

Dent, E. W., Gupton, S. L., \& Gertler, F. B. (2011). The growth cone cytoskeleton in axon outgrowth and guidance. Cold Spring Harbor perspectives in biology, 3(3), a001800. doi:10.1101/cshperspect.a001800

Drummond, M. L., Li, M., Tarapore, E., Nguyen, T. T. L., Barouni, B. J., Cruz, S., . . Atwood, S. X. (2018). Actin polymerization controls cilia-mediated signaling. Journal of Cell Biology, 217(9), 3255-3266. doi:10.1083/jcb.201703196

Drummond-Barbosa, D., \& Spradling, A. C. (2001). Stem cells and their progeny respond to nutritional changes during Drosophila oogenesis. Dev Biol, 231(1), 265-278. doi:10.1006/dbio.2000.0135

Etienne-Manneville, S. (2004). Cdc42 - the centre of polarity. Journal of Cell Science, 117(8), 1291. doi:10.1242/jcs.01115

Fadiga, J., \& Nystul, T. G. (2019). The follicle epithelium in the Drosophila ovary is maintained by a small number of stem cells. eLife, 8, e49050. doi:10.7554/eLife.49050

Flynn, Kevin C., Hellal, F., Neukirchen, D., Jacob, S., Tahirovic, S., Dupraz, S., . . . Bradke, F. (2012). ADF/Cofilin-Mediated Actin Retrograde Flow Directs Neurite Formation in the Developing Brain. Neuron, 76(6), 1091-1107. doi:https://doi.org/10.1016/j.neuron.2012.09.038

Forbes, A. J., Lin, H., Ingham, P. W., \& Spradling, A. C. (1996). hedgehog is required for the proliferation and specification of ovarian somatic cells prior to egg chamber formation in Drosophila. Development, 122(4), 1125-1135. 
Fuwa, T. J., Kinoshita, T., Nishida, H., \& Nishihara, S. (2015). Reduction of T antigen causes loss of hematopoietic progenitors in Drosophila through the inhibition of filopodial extensions from the hematopoietic niche. Dev Biol, 401(2), 206-219. doi:10.1016/j.ydbio.2015.03.003

Gallo, G. (2011). The cytoskeletal and signaling mechanisms of axon collateral branching. Developmental Neurobiology, 71(3), 201-220. doi:10.1002/dneu.20852

González-Méndez, L., Gradilla, A.-C., \& Guerrero, I. (2019). The cytoneme connection: direct longdistance signal transfer during development. Development, 146(9), dev174607. doi:10.1242/dev.174607

Goode, S., Melnick, M., Chou, T. B., \& Perrimon, N. (1996). The neurogenic genes egghead and brainiac define a novel signaling pathway essential for epithelial morphogenesis during Drosophila oogenesis. Development, 122(12), 3863. Retrieved from http://dev.biologists.org/content/122/12/3863.abstract

Goodell, M. A., \& Rando, T. A. (2015). Stem cells and healthy aging. Science, 350(6265), 1199. doi:10.1126/science.aab3388

Greulich, P., \& Simons, B. D. (2016). Dynamic heterogeneity as a strategy of stem cell self-renewal. Proceedings of the National Academy of Sciences, 113(27), 7509. doi:10.1073/pnas.1602779113

Haas, S., Trumpp, A., \& Milsom, M. D. (2018). Causes and Consequences of Hematopoietic Stem Cell Heterogeneity. Cell Stem Cell, 22(5), 627-638. doi:10.1016/j.stem.2018.04.003

Hall, A., \& Lalli, G. (2010). Rho and Ras GTPases in axon growth, guidance, and branching. Cold Spring Harbor perspectives in biology, 2(2), a001818-a001818. doi:10.1101/cshperspect.a001818

Hartman, T. R., Strochlic, T. I., Ji, Y., Zinshteyn, D., \& O'Reilly, A. M. (2013). Diet controls Drosophila follicle stem cell proliferation via Hedgehog sequestration and release. J Cell Biol, 201(5), 741-757. doi:10.1083/jcb.201212094

Hartman, T. R., Ventresca, E. M., Hopkins, A., Zinshteyn, D., Singh, T., O'Brien, J. A., . . O'Reilly, A. M. (2015). Novel tools for genetic manipulation of follicle stem cells in the Drosophila ovary reveal an integrin-dependent transition from quiescence to proliferation. Genetics, 199(4), 935-957. doi:10.1534/genetics.114.173617

Hartman, T. R., Zinshteyn, D., Schofield, H. K., Nicolas, E., Okada, A., \& O'Reilly, A. M. (2010). Drosophila Boi limits Hedgehog levels to suppress follicle stem cell proliferation. J Cell Biol, 191(5), 943-952. doi:10.1083/jcb.201007142

Hinnant, T. D., Merkle, J. A., \& Ables, E. T. (2020). Coordinating Proliferation, Polarity, and Cell Fate in the Drosophila Female Germline. Frontiers in Cell and Developmental Biology, 8(19). doi:10.3389/fcell.2020.00019

Hsu, T.-H., Yang, C.-Y., Yeh, T.-H., Huang, Y.-C., Wang, T.-W., \& Yu, J.-Y. (2017). The Hippo pathway acts downstream of the Hedgehog signaling to regulate follicle stem cell maintenance in the Drosophila ovary. Scientific Reports, 7(1), 4480. doi:10.1038/s41598-017-04052-6

Inaba, M., Buszczak, M., \& Yamashita, Y. M. (2015). Nanotubes mediate niche-stem-cell signalling in the Drosophila testis. Nature, 523(7560), 329-332. doi:10.1038/nature14602

Jevitt, A., Chatterjee, D., Xie, G., Wang, X.-F., Otwell, T., Huang, Y.-C., \& Deng, W.-M. (2020). A single-cell atlas of adult Drosophila ovary identifies transcriptional programs and somatic cell lineage regulating oogenesis. PLOS Biology, 18(4), e3000538. doi:10.1371/journal.pbio.3000538

Jimenez, J., Alphey, L., Nurse, P., \& Glover, D. M. (1990). Complementation of fission yeast cdc2ts and cdc25ts mutants identifies two cell cycle genes from Drosophila: a cdc2 homologue and string. Embo J, 9(11), 3565-3571. 
Jin, Z., Kirilly, D., Weng, C., Kawase, E., Song, X., Smith, S., . . Xie, T. (2008). Differentiation-defective stem cells outcompete normal stem cells for niche occupancy in the Drosophila ovary. Cell Stem Cell, 2(1), 39-49. doi:10.1016/j.stem.2007.10.021

Junyent, S., Garcin, C. L., Szczerkowski, J. L. A., Trieu, T.-J., Reeves, J., \& Habib, S. J. (2020). Specialized cytonemes induce self-organization of stem cells. Proceedings of the National Academy of Sciences, 117(13), 7236. doi:10.1073/pnas.1920837117

Kirilly, D., Spana, E. P., Perrimon, N., Padgett, R. W., \& Xie, T. (2005). BMP signaling is required for controlling somatic stem cell self-renewal in the Drosophila ovary. Dev Cell, 9(5), 651-662. doi:10.1016/j.devcel.2005.09.013

Kronen, M. R., Schoenfelder, K. P., Klein, A. M., \& Nystul, T. G. (2014). Basolateral junction proteins regulate competition for the follicle stem cell niche in the Drosophila ovary. PLoS One, 9(7), e101085. doi:10.1371/journal.pone.0101085

Kunda, P., Paglini, G., Quiroga, S., Kosik, K., \& Caceres, A. (2001). Evidence for the involvement of Tiam1 in axon formation. The Journal of neuroscience : the official journal of the Society for Neuroscience, 21(7), 2361-2372. doi:10.1523/JNEUROSCI.21-07-02361.2001

Laws, K. M., \& Drummond-Barbosa, D. (2016). AMP-activated protein kinase has diet-dependent and independent roles in Drosophila oogenesis. Dev Biol, 420(1), 90-99. doi:10.1016/j.ydbio.2016.10.006

Li, X., Han, Y., \& Xi, R. (2010). Polycomb group genes Psc and Su(z)2 restrict follicle stem cell self-renewal and extrusion by controlling canonical and noncanonical Wnt signaling. Genes Dev, 24(9), 933946. doi:10.1101/gad.1901510

Maes, T., Barceló, A., \& Buesa, C. (2002). Neuron navigator: a human gene family with homology to unc53, a cell guidance gene from Caenorhabditis elegans. Genomics, 80(1), 21-30. doi:10.1006/geno.2002.6799

Margolis, J., \& Spradling, A. (1995). Identification and behavior of epithelial stem cells in the Drosophila ovary. Development, 121(11), 3797-3807.

Merrill, R. A., Plum, L. A., Kaiser, M. E., \& Clagett-Dame, M. (2002). A mammalian homolog of unc-53 is regulated by all-trans retinoic acid in neuroblastoma cells and embryos. Proceedings of the National Academy of Sciences of the United States of America, 99(6), 3422-3427. doi:10.1073/pnas.052017399

Mertens, A. E., Pegtel, D. M., \& Collard, J. G. (2006). Tiam1 takes PARt in cell polarity. Trends Cell Biol, 16(6), 308-316. doi:10.1016/j.tcb.2006.04.001

Nelson, J. O., Chen, C., \& Yamashita, Y. M. (2019). Chapter Six - Germline stem cell homeostasis. In R. Lehmann (Ed.), Current Topics in Developmental Biology (Vol. 135, pp. 203-244): Academic Press.

$\mathrm{Ng}$, J., \& Luo, L. (2004). Rho GTPases regulate axon growth through convergent and divergent signaling pathways. Neuron, 44(5), 779-793. doi:10.1016/j.neuron.2004.11.014

Ng, J., \& Luo, L. (2004). Rho GTPases Regulate Axon Growth through Convergent and Divergent Signaling Pathways. Neuron, 44(5), 779-793. doi:https://doi.org/10.1016/j.neuron.2004.11.014

Nystul, T., \& Spradling, A. (2007). An epithelial niche in the Drosophila ovary undergoes long-range stem cell replacement. Cell Stem Cell, 1(3), 277-285. doi:10.1016/j.stem.2007.07.009

Nystul, T., \& Spradling, A. (2010). Regulation of epithelial stem cell replacement and follicle formation in the Drosophila ovary. Genetics, 184(2), 503-515. doi:10.1534/genetics.109.109538

O'Reilly, A. M., Lee, H. H., \& Simon, M. A. (2008). Integrins control the positioning and proliferation of follicle stem cells in the Drosophila ovary. J Cell Biol, 182(4), 801-815. doi:10.1083/jcb.200710141 
Patel, N. H., Snow, P. M., \& Goodman, C. S. (1987). Characterization and cloning of fasciclin III: a glycoprotein expressed on a subset of neurons and axon pathways in Drosophila. Cell, 48(6), 975988. doi:10.1016/0092-8674(87)90706-9

Potter, C. J., \& Luo, L. (2010). Splinkerette PCR for mapping transposable elements in Drosophila. PLoS One, 5(4), e10168. doi:10.1371/journal.pone.0010168

Reilein, A., Melamed, D., Park, K. S., Berg, A., Cimetta, E., Tandon, N., . . Kalderon, D. (2017). Alternative direct stem cell derivatives defined by stem cell location and graded Wnt signalling. Nat Cell Biol, 19(5), 433-444. doi:10.1038/ncb3505

Reilein, A., Melamed, D., Tavaré, S., \& Kalderon, D. (2018). Division-independent differentiation mandates proliferative competition among stem cells. Proc Natl Acad Sci U S A, 115(14), E3182e3191. doi:10.1073/pnas.1718646115

Rojas-Ríos, P., Guerrero, I., \& González-Reyes, A. (2012). Cytoneme-Mediated Delivery of Hedgehog Regulates the Expression of Bone Morphogenetic Proteins to Maintain Germline Stem Cells in Drosophila. PLOS Biology, 10(4), e1001298. doi:10.1371/journal.pbio.1001298

Ruohola, H., Bremer, K. A., Baker, D., Swedlow, J. R., Jan, L. Y., \& Jan, Y. N. (1991). Role of neurogenic genes in establishment of follicle cell fate and oocyte polarity during oogenesis in Drosophila. Cell, 66(3), 433-449. doi:10.1016/0092-8674(81)90008-8

Rust, K., \& Nystul, T. (2020). Signal transduction in the early Drosophila follicle stem cell lineage. Curr Opin Insect Sci, 37, 39-48. doi:10.1016/j.cois.2019.11.005

Sasaki, N., Kurisu, J., \& Kengaku, M. (2010). Sonic hedgehog signaling regulates actin cytoskeleton via Tiam1-Rac1 cascade during spine formation. Mol Cell Neurosci, 45(4), 335-344. doi:10.1016/j.mcn.2010.07.006

Schmidt, K. L., Marcus-Gueret, N., Adeleye, A., Webber, J., Baillie, D., \& Stringham, E. G. (2009). The cell migration molecule UNC-53/NAV2 is linked to the ARP2/3 complex by ABI-1. Development, 136(4), 563. doi:10.1242/dev.016816

Schultz, M. B., \& Sinclair, D. A. (2016). When stem cells grow old: phenotypes and mechanisms of stem cell aging. Development (Cambridge, England), 143(1), 3-14. doi:10.1242/dev.130633

Singh, T., Lee, E. H., Hartman, T. R., Ruiz-Whalen, D. M., \& O'Reilly, A. M. (2018). Opposing Action of Hedgehog and Insulin Signaling Balances Proliferation and Autophagy to Determine Follicle Stem Cell Lifespan. Dev Cell, 46(6), 720-734 e726. doi:10.1016/j.devcel.2018.08.008

Slaidina, M., Banisch, T. U., Gupta, S., \& Lehmann, R. (2020). A single-cell atlas of the developing Drosophila ovary identifies follicle stem cell progenitors. Genes \& Development. doi:10.1101/gad.330464.119

Smith, J. E., Cummings, C. A., \& Cronmiller, C. (2002). daughterless coordinates somatic cell proliferation, differentiation and germline cyst survival during follicle formation in \&lt;em\&gt;Drosophila\&lt;/em\&gt. Development, 129(13), 3255. Retrieved from http://dev.biologists.org/content/129/13/3255.abstract

Snippert, H. J., van der Flier, L. G., Sato, T., van Es, J. H., van den Born, M., Kroon-Veenboer, C., ... Clevers, H. (2010). Intestinal crypt homeostasis results from neutral competition between symmetrically dividing Lgr5 stem cells. Cell, 143(1), 134-144. doi:10.1016/j.cell.2010.09.016

Snyder, J. C., Rochelle, L. K., Marion, S., Lyerly, H. K., Barak, L. S., \& Caron, M. G. (2015). Lgr4 and Lgr5 drive the formation of long actin-rich cytoneme-like membrane protrusions. Journal of Cell Science, 128(6), 1230. doi:10.1242/jcs.166322 
Sone, M., Hoshino, M., Suzuki, E., Kuroda, S., Kaibuchi, K., Nakagoshi, H., . . Hama, C. (1997). Still life, a protein in synaptic terminals of Drosophila homologous to GDP-GTP exchangers. Science, 275(5299), 543-547. doi:10.1126/science.275.5299.543

Song, X., \& Xie, T. (2002). DE-cadherin-mediated cell adhesion is essential for maintaining somatic stem cells in the Drosophila ovary. Proc Natl Acad Sci U S A, 99(23), 14813-14818. doi:10.1073/pnas.232389399

Song, X., \& Xie, T. (2003). Wingless signaling regulates the maintenance of ovarian somatic stem cells in Drosophila. Development, 130(14), 3259-3268. doi:10.1242/dev.00524

Spehar, K., Pan, A., \& Beerman, I. (2020). Restoring aged stem cell functionality: Current progress and future directions. Stem cells (Dayton, Ohio), 38(9), 1060-1077. doi:10.1002/stem.3234

Su, T. Y., Nakato, E., Choi, P. Y., \& Nakato, H. (2018). Drosophila Glypicans Regulate Follicle Stem Cell Maintenance and Niche Competition. Genetics, 209(2), 537-549. doi:10.1534/genetics.118.300839

Sudarsanam, S., Yaniv, S., Meltzer, H., \& Schuldiner, O. (2020). Cofilin regulates axon growth and branching of Drosophila $p$-neurons. Journal of Cell Science, 133(8), jcs232595. doi:10.1242/jcs.232595

Tanentzapf, G., Smith, C., McGlade, J., \& Tepass, U. (2000). Apical, lateral, and basal polarization cues contribute to the development of the follicular epithelium during Drosophila oogenesis. The Journal of cell biology, 151(4), 891-904. doi:10.1083/jcb.151.4.891

Ulmschneider, B., Grillo-Hill, B. K., Benitez, M., Azimova, D. R., Barber, D. L., \& Nystul, T. G. (2016). Increased intracellular $\mathrm{pH}$ is necessary for adult epithelial and embryonic stem cell differentiation. J Cell Biol, 215(3), 345-355. doi:10.1083/jcb.201606042

Urbán, N., Blomfield, I. M., \& Guillemot, F. (2019). Quiescence of Adult Mammalian Neural Stem Cells: A Highly Regulated Rest. Neuron, 104(5), 834-848. doi:10.1016/j.neuron.2019.09.026

van Velthoven, C. T. J., \& Rando, T. A. (2019). Stem Cell Quiescence: Dynamism, Restraint, and Cellular Idling. Cell Stem Cell, 24(2), 213-225. doi:10.1016/j.stem.2019.01.001

Vied, C., \& Kalderon, D. (2009). Hedgehog-stimulated stem cells depend on non-canonical activity of the Notch co-activator Mastermind. Development (Cambridge, England), 136(13), 2177-2186. doi:10.1242/dev.035329

Vied, C., Reilein, A., Field, N. S., \& Kalderon, D. (2012). Regulation of stem cells by intersecting gradients of long-range niche signals. Dev Cell, 23(4), 836-848. doi:10.1016/j.devcel.2012.09.010

Wahlestedt, M., Erlandsson, E., Kristiansen, T., Lu, R., Brakebusch, C., Weissman, I. L., . . . Bryder, D. (2017). Clonal reversal of ageing-associated stem cell lineage bias via a pluripotent intermediate. Nature Communications, 8(1), 14533. doi:10.1038/ncomms14533

Wan, H., Liu, C., Wert, S. E., Xu, W., Liao, Y., Zheng, Y., \& Whitsett, J. A. (2013). CDC42 is required for structural patterning of the lung during development. Dev Biol, 374(1), 46-57. doi:10.1016/j.ydbio.2012.11.030

Wang, X., \& Page-McCaw, A. (2014). A matrix metalloproteinase mediates long-distance attenuation of stem cell proliferation. J Cell Biol, 206(7), 923-936. doi:10.1083/jcb.201403084

Wang, Z. A., Huang, J., \& Kalderon, D. (2012). Drosophila follicle stem cells are regulated by proliferation and niche adhesion as well as mitochondria and ROS. Nature Communications, 3(1), 769. doi:10.1038/ncomms1765

Wang, Z. A., \& Kalderon, D. (2009). Cyclin E-dependent protein kinase activity regulates niche retention of Drosophila ovarian follicle stem cells. Proc Natl Acad Sci U S A, 106(51), 21701-21706. doi:10.1073/pnas.0909272106 
Xin, T., Greco, V., \& Myung, P. (2016). Hardwiring Stem Cell Communication through Tissue Structure. Cell, 164(6), 1212-1225. doi:10.1016/j.cell.2016.02.041

Zhang, Y., \& Kalderon, D. (2000). Regulation of cell proliferation and patterning in Drosophila oogenesis by Hedgehog signaling. Development, 127(10), 2165-2176.

Zhang, Y., \& Kalderon, D. (2001). Hedgehog acts as a somatic stem cell factor in the Drosophila ovary. Nature, 410(6828), 599-604. doi:10.1038/35069099

Zheng, C., Diaz-Cuadros, M., \& Chalfie, M. (2016). GEFs and Rac GTPases control directional specificity of neurite extension along the anterior-posterior axis. Proceedings of the National Academy of Sciences, 113(25), 6973. doi:10.1073/pnas.1607179113 